\title{
High Value Phycotoxins From the Dinoflagellate Prorocentrum
}

\author{
Dolores Camacho-Muñoz ${ }^{1 *}$, Radisti A. Praptiwi², Linda A. Lawton ${ }^{1}$ and \\ Christine Edwards ${ }^{1}$ \\ ${ }^{1}$ School of Pharmacy and Life Sciences, Robert Gordon University, Aberdeen, United Kingdom, ${ }^{2}$ Department \\ of Biotechnology, Universitas Esa Unggul, Jakarta, Indonesia
}

OPEN ACCESS

Edited by:

Wei Zhang,

Flinders University, Australia

Reviewed by:

Bin Wu,

Zhejiang University, China

Carlos Jimenez,

University of A Coruña, Spain

*Correspondence:

Dolores Camacho-Muñoz

I.camacho-munoz@rgu.ac.uk

Specialty section:

This article was submitted to

Marine Biotechnology,

a section of the journal

Frontiers in Marine Science

Received: 07 December 2020

Accepted: 06 May 2021

Published: 02 June 2021

Citation:

Camacho-Muñoz D, Praptiwi RA Lawton LA and Edwards C (2021) High Value Phycotoxins From the

Dinoflagellate Prorocentrum.

Front. Mar. Sci. 8:638739.

doi: 10.3389/fmars.2021.638739
Marine dinoflagellates produce chemically diverse compounds, with a wide range of biological activity (antimicrobial, anticancer, treatment of neurodegenerative disease along with use as biomedical research tools). Chemical diversity is highlighted by their production of molecules such as the saxitoxin family of alkaloids $\left(\mathrm{C}_{10} \mathrm{H}_{17} \mathrm{~N}_{7} \mathrm{O}_{4}-\right.$ $299 \mathrm{~g} / \mathrm{mol})$ to the amphipathic maitotoxin $\left(\mathrm{C}_{164} \mathrm{H}_{256} \mathrm{O}_{68} \mathrm{~S}_{2} \mathrm{Na}_{2}-3,422 \mathrm{~g} / \mathrm{mol}\right)$, representing one of the largest and most complex secondary metabolites characterized. Dinoflagellates, are most well-known for the production of red tides which are frequently toxic, including okadaic acid and related dinophysistoxins, which are tumor promoters. The mode of action for these phycotoxins, is by specific inhibition of protein phosphatases, enzymes essential in regulation of many cellular processes. Hence, these compounds are being used for vital cell regulation studies. However, the availability of useful amounts of these compounds has restricted research. Chemical synthesis of some compounds such as okadaic acid has been investigated, but the complexity of the molecule resulted in many lengthy steps and achieved only a poor yield. The use of naturally occurring phytoplankton has been investigated as a potential source of these compounds, but it has been shown to be unreliable and impractical. The most practical option is large scale culture with down-stream processing/purification which requires specialist facilities and expertise. This review, describes the biotechnological potential of these organisms and the challenges to achieve useful yields of high quality phycotoxins using Prorocentrum spp. as an example to produce okadaic acid.

Keywords: benthic microalgal cultivation, Prorocentrum lima, seawater, nutrient limitation, temperature, irradiance

\section{INTRODUCTION}

Over the past decades, the occurrence of harmful algae blooms (HABs) have increased worldwide (Luckas et al., 2005; Hallegraeff, 2010; Griffith and Gobler, 2020). Climate change and anthropogenic pressures are considered responsible for increased frequency, intensity and broader geographic range. Moreover, increased social and scientific awareness due to their associated negative socioeconomic effects intensified monitoring HABs (phytoplankton and toxins) (Anderson et al., 2012; Farabegoli et al., 2018; Brown et al., 2020).

Abbreviations: ASP, amnesic shellfish poisoning; AZA, azaspiracid; AZP, azaspiracid shellfish poisoning; BTX, brevetoxin; CFP, ciguatera fish poisoning; CTX, ciguatoxin; DA, domoic acid; DSP, diarrhetic shellfish poisoning; DTX, dinophysistoxin; GYM, gymnodimines; GTX, gonyautoxin; HAB, harmful algae bloom; LED, Light-emitting diode; Mbp, megabase pair; MTX, maitotoxin; NEO, neosaxitoxin; NSP, neurotoxic shellfish poisoning; OA, okadaic acid; PBR, photobioreactor; PLTX, palytoxin; PnTX, pinnatoxin; PP1, phosphatase 1; PP2A, phosphatase 2A; PP2B, phosphatase 2B; PSP, paralytic shellfish poisoning; PTX, pectenotoxin; Qt, toxin quota; SPX, spirolide; STX, saxitoxin; YTX, yessotoxins. 
HABs are typically characterized by rapid proliferation and/or high biomass often known as "red tides" due to their common red color but this color can vary depending on the dominant phytoplankton species involved as well as its concentration and depth. Many of these bloom-forming microalgae produce secondary metabolites, which are toxic (phycotoxins). The purpose of the synthesis of phycotoxins is not clear but it is believed that they may have a role in increasing survival in unfavorable environmental conditions and as a defensive mechanism by inhibiting the growth of co-occurring microalgae or as anti- grazing (Selander et al., 2006; Ajuzie, 2007; Ianora et al., 2011; Anderson et al., 2012; Kohli et al., 2016). Recently, it has also been hypothesized that they could be linked to another trait or process such as photosynthesis II and nitrogen and carbon metabolism (Cassell et al., 2015; Glibert et al., 2016). Nevertheless, the issue arises when phycotoxins bioaccumulate in aquatic edible organisms such as fish and shellfish, leading to eventual poisoning of animal and human consumers (James et al., 2010; Hinder et al., 2011). It is estimated that marine phycotoxins are responsible for up to 60,000 human intoxications per year, with an overall mortality of around $1.5 \%$ (Kantiani et al., 2010).

Based on their chemical structure marine phycotoxins are classified into the following groups: (i) linear and macrocyclic polyethers (e.g., okadaic acid (OA) and dinophysistoxins (DTXs)); (ii) ladder-frame polyethers (e.g., ciguatoxins (CTXs) and brevetoxins (BTXs)); (iii) macrocyclic imines (e.g., spirolides (SPXs) and gymnodimines (GYMs)); (iv) tetrahydropurines (e.g., saxitoxins (STXs) and gonyautoxins (GTXs)); and (v) toxic secondary amines (e.g., domoic acid (DA)). Historically, marine phycotoxins were categorized according to the characteristic symptoms they generate in humans after the consumption of contaminated seafood: diarrhetic shellfish poisoning (DSP) (e.g., OA, DTXs, pectenotoxins (PTXs) and yessotoxins (YTXs)), amnesic shellfish poisoning (ASP) (e.g., DA), paralytic shellfish poisoning (PSP) (e.g., STXs and analogs), azaspiracid shellfish poisoning (AZP) (e.g., azaspiracids (AZAs)), neurotoxic shellfish poisoning (NSP) (e.g., BTXs) and ciguatera fish poisoning (CFP) (e.g., CTX and maitotoxin (MTX)).

This review will focus on the dinoflagellate Prorocentrum spp. as a potential source of phycotoxins and different biotechnological approaches to enhance their production. We aim to summarize the studies conducted on Prorocentrum spp. to enhance the growth rate and DSP toxin production with special emphasis on the influence of nutritional and environmental factors published between 1993 and 2020. It also presents considerations relating to mass cultivation to attain high phycotoxin yields scaling up photobioreactors from bench to large scale.

\section{TOXIC BIOACTIVE METABOLITES FROM DINOFLAGELLATES}

Dinoflagellates are a lineage of unicellular eukaryotes of huge ecological and evolutionary significance (Hoppenrath, 2017;
Cousseau et al., 2020). Approximately half of the dinoflagellate species are autotrophic possessing chloroplasts for photosynthesis, but they are also able to grow heterotrophically, parasitically, mixotrophically or symbiotically (Hoppenrath, 2017; Cousseau et al., 2020). Most dinoflagellates are pelagic but many are adapted to benthic habitats (Gómez, 2012; Durán-Riveroll et al., 2019). Dinoflagellates represent around $75 \%$ of the toxic species responsible for HABs (Smayda, 1997; Cembella, 2003).

Dinoflagellates are known to produce an array of bioactive compounds that are structurally and functionally diverse with valuable toxicological and biological properties (GarciaCamacho et al., 2007). Most of the toxic secondary metabolites are of polyketide origin, including polycyclic polyketides (e.g., YTXs, CTXs, BTXs, MTXs, and palytoxins (PLTXs)), macrolides (e.g., PTXs and amphidinolides) and linear polyketides (e.g., DTXs and OA) (Verma et al., 2019); but also cyclic imines (e.g., SPXs, GYMs and pinnatoxins (PnTXs)) and alkaloids (e.g., STX, GTX, neosaxitoxin (NEO)). Numerous pharmacological functions, such as analgesic, anesthetic, anticancer, antifungal, anticholesterol, cytotoxic, immunesuppressive and/or neurological disease therapeutics were also attributed to these compounds (Garcia-Camacho et al., 2007; Qian et al., 2015; Assunção et al., 2017). Besides their therapeutic use, they are invaluable tools to study cellular processes but their toxicity is a barrier to reaching clinical studies (Cruz et al., 2013; Assunção et al., 2017).

\section{Toxic Bioactive Metabolites From Prorocentrum spp.}

To date, there are approximately 60 species of the dinoflagellate genera Prorocentrum distributed worldwide, half of them in benthic marine habitats (Hoppenrath et al., 2013; Durán-Riveroll et al., 2019). Prorocentrum is known to produce DSP toxins and OA, a major DSP polyketide toxin, hence they are a common chemotaxonomic marker of the toxic Prorocentrum spp. (Fernández et al., 2003; Hoppenrath et al., 2013; Luo et al., 2017; Pan et al., 2017; Nishimura et al., 2020). For example, all reported Prorocentrum lima strains produce OA and some strains also produce DTX1 as a minor toxin (Jackson et al., 1993; McLachlan et al., 1994; Vanucci et al., 2010; Varkitzi et al., 2010; Hoppenrath et al., 2013; Wang et al., 2015; Hou et al., 2016; Lee et al., 2016; Hoppenrath, 2017; Pan et al., 2017; AquinoCruz et al., 2018; Gu et al., 2019). Prorocentrum belizeanum, caipirignum, concavum, maculosum and hoffmannianum are also known producers of DSP toxins (Morton et al., 1994; Zhou and Fritz, 1994; Hoppenrath et al., 2013; López-Rosales et al., 2014; Luo et al., 2017; Accoroni et al., 2018; Rodríguez et al., 2018; Lim et al., 2019; Lee et al., 2020).

OA and DTXs cause gastrointestinal distress with diarrhea, nausea, vomiting and abdominal pain described as the main symptoms (James et al., 2010). They are potent selective inhibitors of protein phosphatases 2A (PP2A), 1 (PP1) and 2B (PP2B) (Bialojan and Takai, 1988; Twiner et al., 2016). It was previously reported that the diarrheic effect of DSP toxins was associated to the inhibitory activity on protein phosphatases 
(Cohen et al., 1990) but no links have been found and their mechanisms of toxicity must be re-evaluated (Munday, 2013). Recent reports point to modulation of neurotransmitters that regulate intestinal mobility, along with water and electrolyte secretion as the cause of DSP syndrome (Valdiglesias et al., 2013; Louzao et al., 2015). Protein phosphatases modulate cell signaling pathways so their inhibition by OA and DTXs renders them as an important tool in medical and physiological studies (Meštrović and Pavela-Vrančič, 2003; Cruz et al., 2013; Valdiglesias et al., 2013). In addition, chronic exposure to OA can induce cytotoxicity, neurotoxicity, immunotoxicity, embriotoxicity, genotoxicity and cancer promotion as reviewed by Vilariño et al. (2018) and Fu et al. (2019). The structurally related compounds, DTXs, share the similar negative effects of OA (Vilariño et al., 2018).

Besides OA and DTXs, other phycotoxins have been identified from cultures of Prorocentrum. For example in P. lima cultures, OA and DTX analogs, as well as formosalides, prorocentrolides and related macrolides, limaol and other non-characterized toxins have been reported (Table 1), however, their significance and biosynthesis have not yet been explored. Prorocentrolides and related macrolides are minor but unique constituents of $P$. lima and they should be considered chemotaxonomic markers (Torigoe et al., 1988; Lu and Chou, 2002; Lee et al., 2019; Li et al., 2020). Prorocentrolides are known as "fastacting toxins" and have been reported to act on nicotinic acetylcholine receptors (Molgó et al., 2017; Amar et al., 2018). Prorocentrolide C exhibited cytotoxicity against cancer cells in vitro (HCT-116 and Neuro-2a cells) (Lee et al., 2019) as well as 4-hydroxyprorocentrolide (inhibitory activity against human colon adenocarcinoma DLD-1 and human malignant melanoma RP-MI7951) (Lu et al., 2005). Formosalides and limaol has also shown cytotoxicity activity against tumor cell lines (hepatocellular carcinoma, colon adenocarcinoma, neuroblastoma and T-cell acute lymphoblastic leukemia cells) (Lu et al., 2009; Yang et al., 2017).

\section{BIOTECHNOLOGICAL APPROACHES FOR PHYCOTOXIN PRODUCTION}

Despite the potential biological activity of these high-value secondary metabolites, only a few have been produced for research and commercial applications. Currently, commercial availability of phycotoxin standards is low and the cost prices are high depending on purity and supplier (e.g.: $\approx 1000-23000$ $€ / \mathrm{mg}$ of $\mathrm{OA} ; \approx 36000-296000 € / \mathrm{mg}$ of $\mathrm{DTX} 1 ; \approx 75000-$ $667000 € / \mathrm{mg}$ of DTX2). Often, the phycotoxin is present in trace amounts in the producer organism, thus a sustainable supply from dinoflagellate harvesting is not feasible.

Among the different approaches for sustainable phycotoxin production are chemical synthesis, harvesting of natural blooms and large-scale, in situ, in vitro and transgenic cultivation of the producer organism. The choice of one or another will depend, among other variables, on the structural complexity of the phycotoxin, the nature of the source and its abundance and the biosynthetic pathway.

\section{Chemical Synthesis}

Chemical synthesis of some phycotoxins and/or characteristic fragments from dinoflagellates has been attempted (Forsyth et al., 1997; Ley et al., 1998; Dounay et al., 1999; Nicolaou et al., 2006; O'Connor and Brimble, 2007; Crimmins et al., 2009; Fürstner et al., 2009; Pang et al., 2011; Stivala et al., 2012; Wilde et al., 2012; Valot et al., 2015). This approach can be unsuitable as it often involves many steps with low yield, and their extremely complex structures make synthesis unfeasible (Nicholas and Phillips, 2006; Wilde et al., 2012). For example, PLTX contains 64 stereogenic centers and its synthesis involved assembly of seven building blocks in 39 steps, requiring a total of more than 140 steps (Armstrong et al., 1989). The synthesis of some phycotoxins, such as OA, has been pursued within the synthetic community due to the potential broad range of biological activities that OA possesses (Isobe et al., 1986; Forsyth et al., 1997; Ley et al., 1998). Successful total synthesis was also reported for 7-deoxy-okadaic acid (Dounay et al., 1999), DTX2 and 2-epiDTX2 (Pang et al., 2011). Chemical synthesis has also supported the structural elucidation of several complex phycotoxins as well as help to deduce their mode of action (Usami, 2009; Wilde et al., 2012).

\section{Genetic Engineering}

Genetic manipulation and overexpression of specific genes associated with the biosynthesis of phycotoxins to enhance their production within the organism is an attractive approach. In order to genetically manipulate dinoflagellates to increase the production of the metabolites of interest it is essential to understand the biochemical processes occurring within the cells and a genome-scale metabolic model could help (Verma et al., 2019). However, the current level of their functional information is poorly understood which may in part be due to the long and complex genome sequences of microalgae. For example dinoflagellates genome (3-250 pg DNA/cell and 3000-215000 megabase pair (Mbp) in 20-325 chromosomes) is approximately 100 times larger than the human genome (3.2 pg DNA/cell and $3180 \mathrm{Mbp}$ in 23 pairs of chromosomes) (Bhaud et al., 2000; McEwan et al., 2008; Verma et al., 2019). Dinoflagellates genome has a high number of unusual bases with a high degree of methylation, introns, redundant repetitive non-coding sequences, chromosomes are organized into a permanent liquid crystalline form (Rill et al., 1989) and it lacks recognizable promoter features (Guillebault et al., 2002) and common eukaryotic transcription factor binding sites (Bhaud et al., 2000; Moreno Díaz de la Espina et al., 2005; McEwan et al., 2008; Wisecaver and Hackett, 2011). Therefore, genetic manipulation methods used for similar or higher trophic systems cannot be extrapolated to dinoflagellates and it must be developed almost from the start. In addition, the identification of toxin-related genes and proteins is complex as a result of their evolutionary origin (Lin, 2011; Verma et al., 2019). To date, most of the efforts on genetic engineering have been driven toward the increase of lipid production in nondinoflagellate algae (Lin et al., 2019; Park et al., 2019; Ng et al., 2020). 
TABLE 1 | High value secondary metabolites produced by Prorocentrum lima.

\section{Bioactive compound}

$\mathrm{OA}$

\section{Microalgae}

P. lima (712); (CCAP 1136/11); (Hainan Island); (Cuba isolates); (Paranagua isolates); (Recife isolates); (Spain isolates); (Tahiti isolates); (UK isolates); (Mexico isolates); (New Zealand); (PL11); (PLV2); (Japan isolates)

\begin{tabular}{|c|c|c|}
\hline & & Tarazona-Janampa et al., 2020 \\
\hline 2-deoxy OA & P. lima & Schmitz and Yasumoto, 1991 \\
\hline 7-deoxy OA & P. lima & $\begin{array}{l}\text { Schmitz and Yasumoto, 1991; Holmes } \\
\text { et al., } 2001\end{array}$ \\
\hline C4-diol OA/OA ester (2-hydroxymethyl-allyl okadaate) & P. lima (PLV2) & Fernández et al., 2003; Paz et al., 2007 \\
\hline C6-diol OA/OA ester (5-hydroxy-2-methylene-pent-3-enyl okadaate) & P. lima & $\begin{array}{l}\text { Suárez-Gómez et al., 2005; Paz et al., } \\
2007\end{array}$ \\
\hline C7-diol OAVOA ester (5-methylene-6-hydroxy-2-hexen-1-okadaate) & P. lima; (GY-H57) & Yasumoto et al., 1989; Pan et al., 2017 \\
\hline C8-diol OA/OA ester (7-hydroxy-2-methyl-hepta-2,4-dienyl okadaate) & P. lima (712); (Mexico isolates) & $\begin{array}{l}\text { Hu et al., 1992; Lu and Chou, 2002; } \\
\text { Suárez-Gómez et al., 2005; Paz et al., } \\
\text { 2007; Tarazona-Janampa et al., } 2020\end{array}$ \\
\hline C9-diol OA/OA ester (5,7-dihydroxy-2,4-dimethylene-heptyl okadaate) & P. lima (GY-H57) & $\begin{array}{l}\text { Suárez-Gómez et al., 2005; Paz et al., } \\
\text { 2007; Pan et al., } 2017\end{array}$ \\
\hline C9-diol OA/OA ester (5-hydroperoxy-7-hydroxy-2,4-dimethylene-heptyl okadaate) & P. lima (PLV2) & $\begin{array}{l}\text { Suárez-Gómez et al., 2005; Paz et al., } \\
2007\end{array}$ \\
\hline C9-diol OA/OA ester (7-hydroxy-4-methyl-2-methylene-hept-4-enyl okadaate) & P. lima (712) & Hu et al., 1992; Paz et al., 2007 \\
\hline C9-diol OA/OA ester (7-hydroxy-2,4-dimethyl-hepta-2,4-dienyl okadaate) & P. lima & Norte et al., 1994; Pan et al., 2017 \\
\hline C9-diol OAVOA ester (7-hydroxy-4-methyl-2-methylene-hept-4-enyl okadaate) & P. lima & Norte et al., 1994; Paz et al., 2007 \\
\hline C9-diol OA & P. lima & $\begin{array}{l}\text { Yasumoto et al., 1987; Lu and Chou, } \\
2002\end{array}$ \\
\hline C10-diol OAVOA ester (5,7-dihydroxy-2,4-bis(methylene)heptyl okadaate) & $\begin{array}{l}\text { P. lima (GY-H57); (Hainan } \\
\text { Island) }\end{array}$ & Pan et al., 2017; Li et al., 2020 \\
\hline Methyl okadaate/OA Methyl ester/Methyl OA & P. lima (PL11); (PL2V); (712) & $\begin{array}{l}\text { Hu et al., 1992; Lu and Chou, 2002; } \\
\text { Fernández et al., 2003; Paz et al., } \\
\text { 2007; Li et al., } 2020\end{array}$ \\
\hline Ethyl okadaate/OA Ethyl ester/Ethyl OA & P. lima (712) & Hu et al., 1992 \\
\hline OA ester & P. lima (Spain isolates) & Bravo et al., 2001 \\
\hline Norokadanone & P. lima (PL2V); (CCAP 1136/11) & $\begin{array}{l}\text { Fernández et al., 2003; Paz et al., } \\
\text { 2007; Camacho-Muñoz et al., } 2020\end{array}$ \\
\hline DTX1 & $\begin{array}{l}\text { P. lima (CCAP 1136/11); (Cuba } \\
\text { isolates); (Paranagua isolates); } \\
\text { (Spain isolates); (UK isolates); } \\
\text { (Mexico isolates); (PL2V); } \\
\text { (Japan isolates) }\end{array}$ & $\begin{array}{l}\text { Hu et al., 1992; Bravo et al., 2001; Lu } \\
\text { and Chou, 2002; Nascimento et al., } \\
\text { 2005; Paz et al., 2007; Pan et al., } \\
\text { 2017; Moreira-González et al., 2019; } \\
\text { Camacho-Muñoz et al., 2020; } \\
\text { Nishimura et al., 2020; } \\
\text { Tarazona-Janampa et al., } 2020\end{array}$ \\
\hline 35S-DTX1 & P. lima (GY-H57) & $\begin{array}{l}\text { Pan et al., 2017; Camacho-Muñoz } \\
\text { et al., } 2020\end{array}$ \\
\hline DTX1-a & P. lima (CCAP 1136/11) & $\begin{array}{l}\text { Camacho-Muñoz et al., 2020; } \\
\text { Tarazona-Janampa et al., } 2020\end{array}$ \\
\hline DTX2 & P. lima (Spain isolates) & $\begin{array}{l}\text { Hu et al., 1992; Bravo et al., 2001; Pan } \\
\text { et al., } 2017\end{array}$ \\
\hline DTX4 & P. lima (UK isolates) & Hu et al., 1995; Nascimento et al., 2005 \\
\hline DTX6 & P. lima (PL2V) & $\begin{array}{l}\text { Suárez-Gómez et al., 2001; Paz et al., } \\
2007\end{array}$ \\
\hline C8-diol DTX1-a & P. lima (Mexico isolates) & Tarazona-Janampa et al., 2020 \\
\hline C8-diol DTX1 & P. lima (Mexico isolates) & Tarazona-Janampa et al., 2020 \\
\hline C9-diol DTX1 & P. lima (PL-KNUAL-23) & Lee et al., 2015 \\
\hline
\end{tabular}

\section{References}

Murakami et al., 1982; Hu et al., 1992; Rhodes and Syhre, 1995; Bravo et al., 2001; Holmes et al., 2001; Lu and Chou, 2002; Fernández et al., 2003; Nascimento et al., 2005; Paz et al., 2007; Li et al., 2012, 2020; Pan et al., 2017; Moreira-González et al., 2019;

Camacho-Muñoz et al., 2020; Nishimura et al., 2020;

Tarazona-Janampa et al., 2020 Schmitz and Yasumoto, 1991 et al., 200 Suárez-Gómez et al., 2005; Paz et al., 2007

Hu et al., 1992; Lu and Chou, 2002; Suárez-Gómez et al., 2005; Paz et al., Suárez-Gómez et al., 2005; Paz et al., 2007; Pan et al., 2017 2007

Hu et al., 1992; Paz et al., 2007

Norte et al 1994; Paz et al, 2007

Yasumoto et al., 1987; Lu and Chou, 2002

Hu et al., 1992; Lu and Chou, 2002; Fernández et al., 2003; Paz et al. Bravo et al., 2001 Hu et al., 1992; Bravo et al., 2001; Lu 2017: Moreira-González et at. 2019 ; Camacho-Muñoz et al., 2020; Pan et al., 2017; Camacho-Muñoz et al., 202

Hu et al., 1992; Bravo et al., 2001; Pan al., 201 Suárez-Gómez et al., 2001; Paz et al., Tarazona-Janampa et al., 2020 Lee et al., 2015 
TABLE 1 | Continued

\begin{tabular}{lll}
\hline Bioactive compound & Microalgae & References \\
\hline DTX2 ester & P. lima (Spain isolates) & Bravo et al., 2001 \\
\hline DTX4 diol ester & P. lima (UK isolates) & Nascimento et al., 2005 \\
\hline Formosalide A & P. lima (PL040104002) & Lu et al., 2009 \\
\hline Formosalide B & P. lima (PL040104002) & Lu et al., 2009 \\
\hline Limaol & P. lima (Korea isolates) & Yang et al., 2017 \\
\hline Prorocentrin & P. lima (PL021117001 clone) & Lu et al., 2005 \\
\hline Prorocentrolide & P. lima (PL01); (PL11) & Lu and Chou, 2002; Li et al., 2020 \\
\hline Procentrolide 30-sulfate & P. lima & Torigoe, 1990 \\
\hline Prorocentrolide C & P. lima (YD-5) & Lee et al., 2019 \\
\hline 14-O-acetyl-4-hydroxyprorocentrolide & P. lima (PL01) & Lu and Chou, 2002 \\
\hline 3,5-dihydroxy-6,7-megastigmadien-9-one & P. lima (PL11) & Li et al., 2020 \\
\hline 4-hydroxyprorocentrolide & P. lima (PL01); (YD-5) & Lu and Chou, 2002; Lee et al., 2019 \\
\hline 9,51-dihydroprorocentrolide & P. lima & Torigoe, 1990 \\
\hline Apo-9'-fucoxanthinone & P. lima (PL11) & Li et al., 2020 \\
\hline Polyketide unidentified & P. lima (PL11) & Li et al., 2020
\end{tabular}

\section{Culture of Dinoflagellates}

Dinoflagellates in general grow slower than other protists such as diatoms, are quite shear-sensitive and relatively inefficient at nutrient uptake (Smayda and Reynolds, 2003). It has been speculated that the low chlorophyll a to carbon ratio is responsible for the slow growth of dinoflagellates (Tang, 1996) but the chlorophyll a content per unit mass of dinoflagellates is similar to diatoms (Gallardo-Rodríguez et al., 2012). Several studies have focused on small-scale cultures of benthic dinoflagellates to optimize growth rates and toxic production (Jackson et al., 1993; McLachlan et al., 1994; Morton et al., 1994; Yang et al., 2008; Zhong et al., 2008; Li et al., 2009; Vanucci et al., 2010; Varkitzi et al., 2010; López-Rosales et al., 2014; Wang et al., 2015; Hou et al., 2016; Accoroni et al., 2018; Aquino-Cruz et al., 2018; Gu et al., 2019; Lee et al., 2020). In a typical photosynthetic culture the maximum biomass concentration reached was well below $1 \mathrm{~g} / \mathrm{L}$ and regarding phycotoxin production it is of the order of picograms (GallardoRodríguez et al., 2012). Therefore, to obtain sufficient secondary metabolites mass culture in large-scale bioreactors is needed although it has proven challenging (Gallardo Rodríguez et al., 2010; Wang et al., 2015).

Similar to other phototrophic organisms, dinoflagellates require energy from light for their biosynthesis processes. Vertical migration and photo-acclimation allow them to travel and grow at the bottom and the upper layer of the aquatic system to gain access to nutrient-rich deeper layers and well-lit surface waters (Smayda and Reynolds, 2003; Smayda, 2010; Orchard et al., 2016; Shikata et al., 2020).

Typical dinoflagellate cell cycle involves an initial growth phase with high cell metabolic activity first (G1 phase), followed by DNA synthesis (S phase). Then, a second growth phase (G2 phase) in which cells accumulate nutrients needed for the mitosis (M phase) and cytokinesis (Pan et al., 1999; Bhaud et al., 2000). The biosynthesis of phycotoxins typically varies during different growth phases, with the highest production rates during the end of the exponential phase prior entering the stationary phase (Pan et al., 1999; Jia et al., 2019; Lee et al., 2020). Circadian rhythms are also known to influence growth rates and biosynthesis of secondary metabolites (Jacob-Lopes et al., 2009; Jia et al., 2019).

Dinoflagellate tolerance and acclimatization to different temperatures are strain specific and may depend on their original habitat as it was found that dinoflagellates from tropical habitats have lower tolerance to low temperatures (below $20^{\circ} \mathrm{C}$ ) than dinoflagellates from temperate habitats (Jackson et al., 1993; McLachlan et al., 1994; Accoroni et al., 2018).

OA and DTXs are essentially produced via the polyketide pathway (Perez et al., 2008; Gallardo-Rodríguez et al., 2012). Therefore, the interaction between dinoflagellates (i.e., Prorocentrum) and their co-existing microbiome encoding the polyketide synthase gene may influence intracellular phycotoxin production (Perez et al., 2008; Lee et al., 2016). However, to date, there is little evidence that supports that extracellular bacteria influence growth and phycotoxin production (TarazonaJanampa et al., 2020). A sound knowledge of cell cycle regulation and metabolism, synchronization of the cell cycle, use of optimum nutritional requirements and culture conditions, awareness of stress factors and optimized configurations of bioreactors are fundamental to mass culture of dinoflagellates for phycotoxin production.

\section{CULTURE OF Prorocentrum spp. FOR PHYCOTOXIN PRODUCTION}

The effects of key environmental factors (e.g., nutrients, temperature, salinity, and light availability) on the phycotoxin production are species dependent (Vanucci et al., 2010; Varkitzi et al., 2010; Wang et al., 2015; Aquino-Cruz et al., 2018; Gu et al., 2019; Lee et al., 2020). Nutrient limitation is the most common strategy used to boost phycotoxin production, however as a result of nutrient limitation cell growth usually declines. 
Phycotoxin production was deemed to be a dependent of growth rate rather than due to the environmental or nutritional stress applied (Pistocchi et al., 2012). Therefore, a compromise must be reached between high phycotoxin production and maximum growth rate, leading generally to two-step culture strategies, with an initial phase based on biomass accumulation (nutrient replete conditions) followed by a phase of phycotoxin production (under deprivation conditions). Alternative strategies include changes in the light cycle, $\mathrm{pH}$, temperature or salinity to boost phycotoxin production.

\section{Effect of Culture Media Composition Media}

Natural seawater enriched with nutrients and trace metals is frequently used as a base for culture medium of dinoflagellates (Harrison and Berges, 2005). However, its continuous supply may be inconvenient so synthetic seawater is commonly used instead when a natural seawater supply is unfeasible (e.g., geographic limitations). Although composition of synthetic seawater can be easily controlled and modified, trace organics present in natural seawater are difficult to reproduce in its artificial counterpart. L1, L1-Si, f/2, f/4, K medium have been used to enrich the natural or synthetic seawater for the cultivation of Prorocentrum spp. (Tables 2-6). All these media contains similar nutritional elements (nitrogen, phosphorus, sulfates and trace metals and vitamins) but in different ratios. According to Guillard and Morton (2003) L1 and f/2 medium are more suitable for coastal planktonic species, while L2 and $\mathrm{K}$ media are favorable for growing benthic, epiphytic and oceanic planktonic species. Although the use of artificial seawater is acceptable for cultivating marine dinoflagellates, its long term replacement for natural seawater was deemed to lower production of bioactive metabolites (Hsieh et al., 2001). When P. lima was cultured in $f / 2$ media prepared in natural seawater, artificial seawater (Guillard and Morton, 2003), and salt water $(\mathrm{NaCl} 35 \mathrm{~g} / \mathrm{l})$ it was able to grow in all types of prepared medium. However, the use of salt water affected the cell integrity in the culture that was indicated by lack of pigmentation and high levels of OA in the medium (Praptiwi, 2014). Long-term artificial seawater cultivation resulted in a substantial reduction of OA production of almost $70 \%$ after the second cycle of sub-culturing (Praptiwi, 2014) suggesting that artificial seawater may lack trace elements or nutrients present in natural seawater necessary to support phytotoxin production.

\section{Nitrogen}

Microalgae are able to obtain nitrogen from different sources. Benthic Prorocentrum spp. has been cultured using nitrate, ammonium and urea (Table 2) (McLachlan et al., 1994; Pan et al., 1999; Zhong et al., 2008; Li et al., 2009; Varkitzi et al., 2010; Hou et al., 2016; Accoroni et al., 2018; Gu et al., 2019; Lee et al., 2020). Whereas some studies suggest that nitrogen concentration is directly proportional to the maximum cell density but not growth rate (Zhong et al., 2008; Vanucci et al., 2010; Hou et al., 2016), others found that it is directly proportional to both (McLachlan et al., 1994; Li et al., 2009).
Under, routine culture conditions, maximum phycotoxin levels have been recorded at the end of the exponential growth phase and start of the stationary phase (McLachlan et al., 1994; Li et al., 2009; Varkitzi et al., 2010). However, when microalgae is grown under stress conditions, such as nutrient depletion, they synthesize multiple secondary metabolites, phycotoxins among them, to increase the possibility of survival under these unfavorable conditions. In general, limited nitrogen availability leads to an increase in phycotoxin production (McLachlan et al., 1994; Hou et al., 2016; Accoroni et al., 2018; Gu et al., 2019; Lee et al., 2020). For instance, in a nitrogen-depleted medium, intracellular concentration of $\mathrm{OA}$ in $P$. hoffmannianum was 3.7 times basal nitrogen level $(883 \mu \mathrm{M})$ during the stationary phase (45.38 pg/cell) and 3.4 times this during its maximum growth (21.38 pg/cell) (Lee et al., 2020) (Table 2). Whereas in a medium with ten times more nitrogen $(8830 \mu \mathrm{M})$ intracellular concentration of $\mathrm{OA}$ was similar during the stationary and the maximum growth phase (both $\approx 16.38 \mathrm{pg} /$ cell) but the phycotoxin levels were higher in the maximum growth phase (2.6 times) than in the stationary phase (1.3 times) (Lee et al., 2020). Vanucci et al. (2010) demonstrated a similar trend of increased phycotoxin production as the concentration of nitrogen was decreased. In this case the effect was more noticeable in the production of DTX1 than in OA, with DTX1 production increased up to 3.3 times $(0.39 \mathrm{pg} / \mathrm{cell})$ and OA production up to 1.9 times $(12.5 \mathrm{pg} /$ cell) in a culture of $P$. lima when the concentration of nitrogen was reduced to $17.7 \mu \mathrm{M}$ (Table 2) from basal levels. The difference on OA yield observed between $P$. hoffmannianum and $P$. lima when changing $\mathrm{N}$ concentrations could be due to be strain specific or due to the small differences between the culturing conditions.

In addition, the chemical nature of nitrogen may affect the phycotoxin production (Table 2). It has been suggested that ammonia may be a preferred source of nitrogen for benthic Prorocentrum spp. as it does not need enzymatic fixation although it does not seem to increase phycotoxin production (Pan et al., 1999; Zhong et al., 2008; Varkitzi et al., 2010). Gu et al. (2019) compared OA production of $P$. lima cultured with nitrate, urea or ammonium. Maximum cell density (47000 cells $/ \mathrm{mL}$ ) and $\mathrm{OA}$ production $(25 \mathrm{pg} / \mathrm{cell}$ ) were obtained using nitrate as a source and the lowest with ammonium. Similar effects were obtained by Varkitzi et al. (2010) when the same concentration of nitrate or ammonium were used for growing $P$. lima. Zhong et al. (2008) also reported the highest OA production using $100 \mu \mathrm{M}$ nitrates (OA $338 \mathrm{pg} / \mathrm{cell}$ ) but no differences were observed among $100 \mu \mathrm{M}$ urea (OA $113 \mathrm{pg} /$ cell) and $100 \mu \mathrm{M}$ ammonium (OA $100 \mathrm{pg} / \mathrm{cell}$ ). In the study of Gu et al. (2019) the use of different nitrogen sources led to different phycotoxin production, maximum cell densities and growth rates. In general, nitrates gave the maximum OA production and cell density followed by urea and finally ammonium.

\section{Phosphorus}

Phosphorus is an essential nutrient involved in several metabolic processes in dinoflagellates including photosynthesis, cell membrane synthesis, signal transduction and catabolism of sugars and fatty acids, among others (Lin et al., 2016). 
TABLE 2 | Effect of nitrogen and phosphorus on the growth and toxin content of Prorocentrum spp.

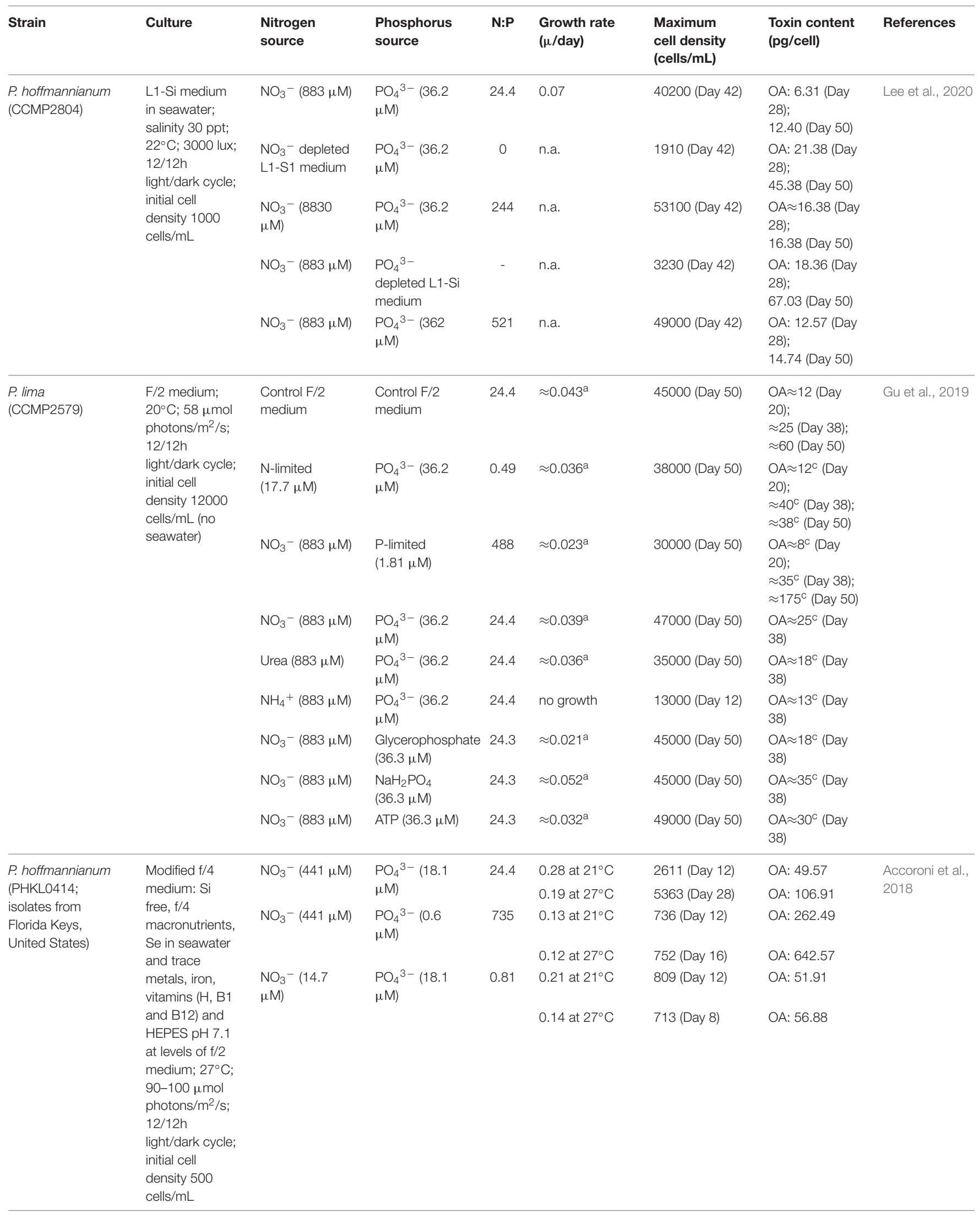


TABLE 2 | Continued

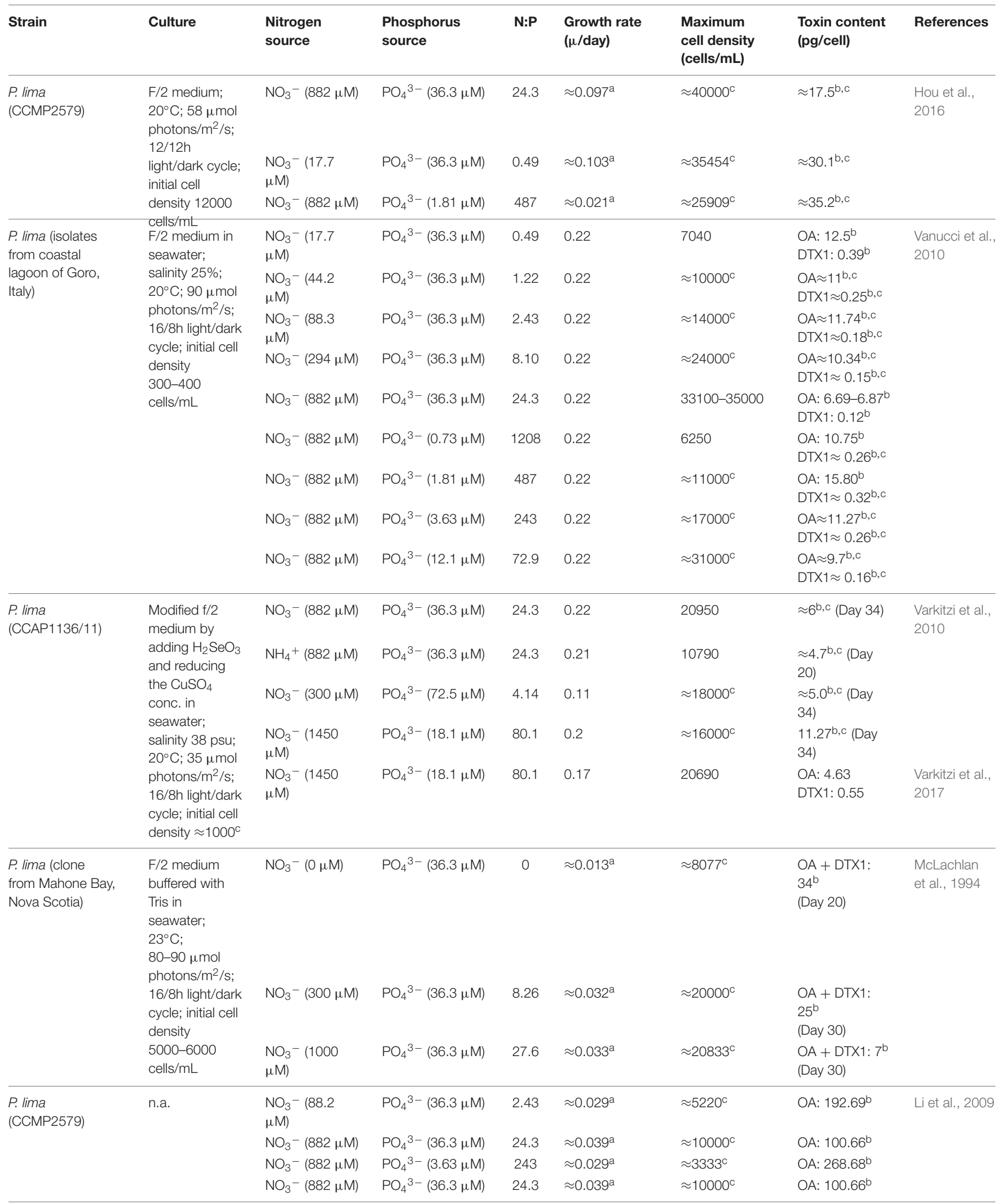


TABLE 2 | Continued

\begin{tabular}{|c|c|c|c|c|c|c|c|c|}
\hline Strain & Culture & $\begin{array}{l}\text { Nitrogen } \\
\text { source }\end{array}$ & $\begin{array}{l}\text { Phosphorus } \\
\text { source }\end{array}$ & $N: P$ & $\begin{array}{l}\text { Growth rate } \\
\text { ( } \mu / \text { day) }\end{array}$ & $\begin{array}{l}\text { Maximum } \\
\text { cell density } \\
\text { (cells/mL) }\end{array}$ & $\begin{array}{l}\text { Toxin content } \\
\text { (pg/cell) }\end{array}$ & References \\
\hline \multirow{12}{*}{$\begin{array}{l}\text { P. lima } \\
\text { (CCMP2579) }\end{array}$} & \multirow[t]{12}{*}{ n.a. } & $\mathrm{NO}_{3}-(12 \mu \mathrm{M})$ & $\mathrm{PO}_{4}{ }^{3-}(36.3 \mu \mathrm{M})$ & 0.33 & 0.058 & $\approx 13180^{\circ}$ & $\mathrm{OA} \approx 210^{\mathrm{b}, \mathrm{c}}$ & \multirow[t]{12}{*}{ Zhong et al., 2008} \\
\hline & & $\mathrm{NO}_{3}-(25 \mu \mathrm{M})$ & $\mathrm{PO}_{4}^{3-}(36.3 \mu \mathrm{M})$ & 0.69 & 0.058 & $\approx 13180^{\circ}$ & $\mathrm{OA} \approx 275^{\mathrm{b}, \mathrm{c}}$ & \\
\hline & & $\mathrm{NO}_{3}-(50 \mu \mathrm{M})$ & $\mathrm{PO}_{4}{ }^{3-}(36.3 \mu \mathrm{M})$ & 1.38 & 0.058 & $\approx 14540^{c}$ & $\mathrm{OA} \approx 240^{\mathrm{b}, \mathrm{c}}$ & \\
\hline & & $\mathrm{NO}_{3}-(100 \mu \mathrm{M})$ & $\mathrm{PO}_{4}^{3-}(36.3 \mu \mathrm{M})$ & 2.75 & 0.058 & $\approx 15000^{\mathrm{C}}$ & $\mathrm{OA} \approx 338^{\mathrm{b}, \mathrm{c}}$ & \\
\hline & & $\mathrm{NH}_{4}(12 \mu \mathrm{M})$ & $\mathrm{PO}_{4}^{3-}(36.3 \mu \mathrm{M})$ & 0.33 & 0.059 & $\approx 13210^{c}$ & $\mathrm{OA} \approx 85^{\mathrm{b}, \mathrm{c}}$ & \\
\hline & & $\mathrm{NH}_{4}(25 \mu \mathrm{M})$ & $\mathrm{PO}_{4}^{3-}(36.3 \mu \mathrm{M})$ & 0.69 & 0.059 & $\approx 11740^{\mathrm{C}}$ & $\mathrm{OA} \approx 68^{\mathrm{b}, \mathrm{c}}$ & \\
\hline & & $\mathrm{NH}_{4}(50 \mu \mathrm{M})$ & $\mathrm{PO}_{4}^{3-}(36.3 \mu \mathrm{M})$ & 1.38 & 0.059 & $\approx 12500^{\mathrm{C}}$ & $\mathrm{OA} \approx 85^{\mathrm{b}, \mathrm{c}}$ & \\
\hline & & $\mathrm{NH}_{4}(100 \mu \mathrm{M})$ & $\mathrm{PO}_{4}{ }^{3-}(36.3 \mu \mathrm{M})$ & 2.75 & 0.059 & $\approx 13620^{c}$ & $\mathrm{OA} \approx 100^{\mathrm{b}, \mathrm{c}}$ & \\
\hline & & Urea (12 $\mu \mathrm{M})$ & $\mathrm{PO}_{4}^{3-}(36.3 \mu \mathrm{M})$ & 0.33 & 0.06 & $\approx 13880^{\circ}$ & $O A \approx 100^{b, c}$ & \\
\hline & & Urea $(25 \mu \mathrm{M})$ & $\mathrm{PO}_{4}{ }^{3-}(36.3 \mu \mathrm{M})$ & 0.69 & 0.06 & $\approx 13330^{c}$ & $\mathrm{OA} \approx 58^{\mathrm{b}, \mathrm{c}}$ & \\
\hline & & Urea $(50 \mu \mathrm{M})$ & $\mathrm{PO}_{4}^{3-}(36.3 \mu \mathrm{M})$ & 1.38 & 0.06 & $\approx 15550^{\mathrm{C}}$ & $\mathrm{OA} \approx 55^{\mathrm{b}, \mathrm{c}}$ & \\
\hline & & Urea $(100 \mu \mathrm{M})$ & $\mathrm{PO}_{4}^{3-}(36.3 \mu \mathrm{M})$ & 2.75 & 0.06 & 18330 & $\mathrm{OA} \approx 113^{\mathrm{b}, \mathrm{c}}$ & \\
\hline \multirow{15}{*}{$\begin{array}{l}\text { P. lima } \\
\text { (CCMP2579) }\end{array}$} & \multirow[t]{15}{*}{ n.a. } & $\mathrm{NO}_{3}-(882 \mu \mathrm{M})$ & $\mathrm{PO}_{4}^{3-}(0.5 \mu \mathrm{M})$ & 1764 & 0.059 & $\approx 12230^{\circ}$ & $\mathrm{OA} \approx 88^{\mathrm{b}, \mathrm{c}}$ & \multirow[t]{15}{*}{ Yang et al., 2008} \\
\hline & & $\mathrm{NO}_{3}-(882 \mu \mathrm{M})$ & $\mathrm{PO}_{4}^{3-}(1 \mu \mathrm{M})$ & 882 & 0.059 & $\approx 13290^{c}$ & $\mathrm{OA} \approx 76^{\mathrm{b}, \mathrm{c}}$ & \\
\hline & & $\mathrm{NO}_{3}-(882 \mu \mathrm{M})$ & $\mathrm{PO}_{4}{ }^{3-}(2 \mu \mathrm{M})$ & 441 & 0.059 & $\approx 14200^{\mathrm{C}}$ & $\mathrm{OA} \approx 86^{\mathrm{b}, \mathrm{c}}$ & \\
\hline & & $\mathrm{NO}_{3}-(882 \mu \mathrm{M})$ & $\mathrm{PO}_{4}{ }^{3-}(5 \mu \mathrm{M})$ & 176 & 0.059 & $\approx 15000^{c}$ & $\mathrm{OA} \approx 60^{\mathrm{b}, \mathrm{c}}$ & \\
\hline & & $\mathrm{NO}_{3}-(882 \mu \mathrm{M})$ & $\mathrm{PO}_{4}{ }^{3-}(10 \mu \mathrm{M})$ & 88.2 & 0.059 & $\approx 16000^{c}$ & $\mathrm{OA} \approx 68^{\mathrm{b}, \mathrm{c}}$ & \\
\hline & & $\mathrm{NO}_{3}-(882 \mu \mathrm{M})$ & $\begin{array}{l}\text { Gly } \mathrm{PO}_{4}^{3-} \\
(0.5 \mu \mathrm{M})\end{array}$ & 1764 & 0.048 & $\approx 11840^{\mathrm{C}}$ & $\mathrm{OA} \approx 116^{\mathrm{b}, \mathrm{c}}$ & \\
\hline & & $\mathrm{NO}_{3}-(882 \mu \mathrm{M})$ & Gly $\mathrm{PO}_{4}{ }^{3-}(1 \mu \mathrm{M})$ & 882 & 0.048 & $\approx 11840^{\mathrm{C}}$ & $\mathrm{OA} \approx 97^{\mathrm{b}, \mathrm{c}}$ & \\
\hline & & $\mathrm{NO}_{3}-(882 \mu \mathrm{M})$ & Gly $\mathrm{PO}_{4}{ }^{3-}(2 \mu \mathrm{M})$ & 441 & 0.048 & $\approx 11440^{\mathrm{C}}$ & $\mathrm{OA} \approx 100^{\mathrm{b}, \mathrm{c}}$ & \\
\hline & & $\mathrm{NO}_{3}-(882 \mu \mathrm{M})$ & Gly $\mathrm{PO}_{4}{ }^{3-}(5 \mu \mathrm{M})$ & 176 & 0.048 & $\approx 13600^{c}$ & $\mathrm{OA} \approx 94^{\mathrm{b}, \mathrm{c}}$ & \\
\hline & & $\mathrm{NO}_{3}-(882 \mu \mathrm{M})$ & Gly $\mathrm{PO}_{4}^{3-}(10 \mu \mathrm{M})$ & 88.2 & 0.048 & $\approx 16310^{\mathrm{C}}$ & $\mathrm{OA} \approx 88^{\mathrm{b}, \mathrm{c}}$ & \\
\hline & & $\mathrm{NO}_{3}-(882 \mu \mathrm{M})$ & ATP $(0.5 \mu \mathrm{M})$ & 1764 & 0.053 & $\approx 13160^{\mathrm{c}}$ & $\mathrm{OA} \approx 51^{\mathrm{b}, \mathrm{c}}$ & \\
\hline & & $\mathrm{NO}_{3}-(882 \mu \mathrm{M})$ & ATP $(1 \mu \mathrm{M})$ & 882 & 0.053 & $\approx 13830^{\circ}$ & $\mathrm{OA} \approx 57^{\mathrm{b}, \mathrm{c}}$ & \\
\hline & & $\mathrm{NO}_{3}-(882 \mu \mathrm{M})$ & ATP $(2 \mu \mathrm{M})$ & 441 & 0.053 & $\approx 17000^{c}$ & $\mathrm{OA} \approx 57^{\mathrm{b}, \mathrm{c}}$ & \\
\hline & & $\mathrm{NO}_{3}-(882 \mu \mathrm{M})$ & ATP $(5 \mu \mathrm{M})$ & 176 & 0.053 & $\approx 18160^{\mathrm{C}}$ & $\mathrm{OA} \approx 60^{\mathrm{b}, \mathrm{c}}$ & \\
\hline & & $\mathrm{NO}_{3}-(882 \mu \mathrm{M})$ & ATP $(10 \mu \mathrm{M})$ & 88.2 & 0.053 & $\approx 19160^{\mathrm{C}}$ & $\mathrm{OA} \approx 57^{\mathrm{b}, \mathrm{c}}$ & \\
\hline
\end{tabular}

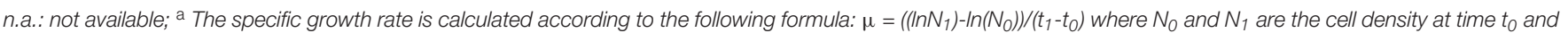

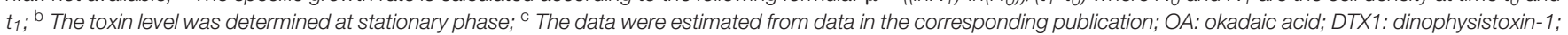
N: nitrogen; $P$ : phosphorus.

Limited availability of phosphorus in culture medium has also proved to hinder the growth of Prorocentrum spp. (Table 2) (Hou et al., 2016; Accoroni et al., 2018; Gu et al., 2019). Whereas production of OA and DTX was promoted when dinoflagellates were grown under P-limitation (Vanucci et al., 2010; Varkitzi et al., 2010; Hou et al., 2016; Accoroni et al., 2018; Lee et al., 2020). In general, studies evaluate the effect of P-depletion on the growth rate, maximum cell density and phycotoxin production but not the effect of P-augmentation. Lee et al. (2020) observed that $362 \mu \mathrm{M}$ of $\mathrm{P}$ had no great impact on the maximum cell density (20\% increase) and phycotoxin production (1.2 times greater) of P. hoffmannianum in comparison to the effect of P-depletion had (maximum cell density was decreased by $92 \%$ but phycotoxin content increased 5.4 times).

The chemical nature of phosphorus also influence growth rates and cellular phycotoxin levels in P. lima (Gu et al., 2019; Yang et al., 2008) (Table 2). Maximum cell growth and $\mathrm{OA}$ production $(35 \mathrm{pg} / \mathrm{cell})$ was observed using sodium dihydrogen phosphate $\left(\mathrm{NaH}_{2} \mathrm{PO}_{4}\right)$, followed by ATP (30 pg/cell) and finally glycerophosphate (18 pg/cell) (Gu et al., 2019).
However, maximum cell density was similar regardless of the phosphorus source used (Gu et al., 2019). Yang et al. (2008) observed a similar effect on the growth rate and the maximum cell density of P. lima after different phosphorus sources were used. However in this case the phosphorus concentrations evaluated $(0.5$ to $10 \mu \mathrm{M})$ were below typical culture media $(36.3 \mu \mathrm{M})$ and the maximum $\mathrm{OA}$ cell content increased as the concentration decreased (from 10 to $0.5 \mu \mathrm{M}$ ) after using glycerophosphate (from $88 \mathrm{pg} / \mathrm{cell}$ to $116 \mathrm{pg} / \mathrm{cell}$ of $\mathrm{OA}$ ), and $\mathrm{NaH}_{2} \mathrm{PO}_{4}$ (from $68 \mathrm{pg} / \mathrm{cell}$ to $88 \mathrm{pg} / \mathrm{cell}$ ) but not after using ATP (from 57 $\mathrm{pg} / \mathrm{cell}$ to $51 \mathrm{pg} / \mathrm{cell}$ ).

\section{Trace Metals}

In microalgae, trace metals play important roles in numerous metabolic processes including electron and oxygen transport, nutrient acquisition, anti-oxidative mechanisms, cell division and integrity (Bruland et al., 1991; Raven et al., 1999; Sunda, 2012). Growth and cell morphology may be altered by a deficiency of trace metals but an excess could induce cell death and cyst formation (Tian et al., 2018). 
TABLE 3 | Effect of light on the growth and toxin content of Prorocentrum spp.

\begin{tabular}{|c|c|c|c|c|c|c|c|}
\hline Strain & Culture & $\begin{array}{c}\text { Light(h)/ } \\
\text { dark(h) } \\
\text { cycle }\end{array}$ & Illuminance & $\begin{array}{l}\text { Growth } \\
\text { rate } \\
(\mu / \text { day })\end{array}$ & $\begin{array}{l}\text { Maximum } \\
\text { cell density } \\
\text { (cells } / \mathrm{mL} \text { ) }\end{array}$ & $\begin{array}{l}\text { Toxin content } \\
\text { (pg/cell) }\end{array}$ & References \\
\hline \multirow[t]{2}{*}{$\begin{array}{l}\text { P. lima } \\
\text { (CCMP 2579) }\end{array}$} & \multirow[t]{2}{*}{$\begin{array}{l}\mathrm{F} / 2 \text { medium; salinity } 30 \% ; \\
25^{\circ} \mathrm{C} ; 100 \mu \mathrm{mol} \text { photons } / \mathrm{m}^{2} / \mathrm{s} ; \\
\text { initial cell density } 5000 \mathrm{cells} / \mathrm{mL}\end{array}$} & $\begin{array}{l}8 / 16 \\
12 / 12\end{array}$ & $\begin{array}{c}100 \mu \mathrm{mol} \\
\text { photons } / \mathrm{m}^{2} / \mathrm{s} \\
100 \mu \mathrm{mol} \\
\text { photons } / \mathrm{m}^{2} / \mathrm{s}\end{array}$ & $\begin{array}{l}\text { n.a. } \\
\text { n.a. }\end{array}$ & $\begin{array}{l}\approx 11000 \text { (Day } \\
35 \text { ) } \\
\approx 25500 \text { (Day } \\
35 \text { ) }\end{array}$ & $\begin{array}{l}O A \approx 1.5^{b, c} \\
O A \approx 1.5^{b, c}\end{array}$ & Wang et al., 2015 \\
\hline & & $16 / 8$ & $\begin{array}{c}100 \mu \mathrm{mol} \\
\text { photons } / \mathrm{m}^{2} / \mathrm{s}\end{array}$ & n.a. & $\begin{array}{l}\approx 26000 \text { (Day } \\
\text { 35) }\end{array}$ & $\mathrm{OA} \approx 1.2^{\mathrm{b}, \mathrm{c}}$ & \\
\hline \multirow{12}{*}{$\begin{array}{l}\text { P. belizeanum } \\
\text { (VGO1029), } \\
\text { Isolates from } \\
\text { La Puntilla, } \\
\text { Las Palmas de } \\
\text { Gran Canaria) }\end{array}$} & \multirow{12}{*}{$\begin{array}{l}\text { L1 medium in seawater; } \\
40 \mu \mathrm{mol} \text { photons } / \mathrm{m}^{2} / \mathrm{s} ; \text { initial } \\
\text { cell density } 10000 \mathrm{cells} / \mathrm{mL}\end{array}$} & $12 / 12$ & $\begin{array}{c}20 \mu \mathrm{mol} \text { photons } / \mathrm{m}^{2} / \mathrm{s} \\
\text { at } 18^{\circ} \mathrm{C}\end{array}$ & $\approx 0.075^{\mathrm{a}, \mathrm{c}}$ & $\begin{array}{c}\approx 60000^{\circ} \text { (Day } \\
25)\end{array}$ & n.a. & $\begin{array}{l}\text { López-Rosales } \\
\text { et al., } 2014\end{array}$ \\
\hline & & $12 / 12$ & $\begin{array}{c}40 \mu \mathrm{mol} \text { photons } / \mathrm{m}^{2} / \mathrm{s} \\
\text { at } 18^{\circ} \mathrm{C}\end{array}$ & $\approx 0.120^{\mathrm{a}, \mathrm{c}}$ & $\begin{array}{c}\approx 120000^{c} \text { (Day } \\
\text { 25) }\end{array}$ & $\mathrm{OA} \approx 4.75^{\mathrm{b}, \mathrm{c}}$ & \\
\hline & & $12 / 12$ & $\begin{array}{c}80 \mu \mathrm{mol} \text { photons } / \mathrm{m}^{2} / \mathrm{s} \\
\text { at } 18^{\circ} \mathrm{C}\end{array}$ & $\approx 0.120^{\mathrm{a}, \mathrm{c}}$ & $\begin{array}{c}\approx 120000^{\circ} \text { (Day } \\
\text { 25) }\end{array}$ & n.a. & \\
\hline & & $12 / 12$ & $\begin{array}{c}120 \mu \mathrm{mol} \\
\text { photons } / \mathrm{m}^{2} / \mathrm{s} \text { at } 18^{\circ} \mathrm{C}\end{array}$ & $\approx 0.105^{a, c}$ & $\begin{array}{c}\approx 80000^{\circ} \text { (Day } \\
25 \text { ) }\end{array}$ & n.a. & \\
\hline & & $12 / 12$ & $\begin{array}{c}20 \mu \mathrm{mol} \text { photons } / \mathrm{m}^{2} / \mathrm{s} \\
\text { at } 25^{\circ} \mathrm{C}\end{array}$ & $\approx 0.070^{a, c}$ & $\begin{array}{c}\approx 35000^{c} \text { (Day } \\
25 \text { ) }\end{array}$ & n.a. & \\
\hline & & $12 / 12$ & $\begin{array}{c}40 \mu \mathrm{mol} \text { photons } / \mathrm{m}^{2} / \mathrm{s} \\
\text { at } 25^{\circ} \mathrm{C}\end{array}$ & $\approx 0.200^{\mathrm{a}, \mathrm{c}}$ & $\begin{array}{l}\approx 135000^{\circ} \text { (Day } \\
20 \text { ) }\end{array}$ & $\mathrm{OA} \approx 1.1^{\mathrm{b}, \mathrm{c}}$ & \\
\hline & & $12 / 12$ & $\begin{array}{c}80 \mu \mathrm{mol} \text { photons } / \mathrm{m}^{2} / \mathrm{s} \\
\text { at } 25^{\circ} \mathrm{C}\end{array}$ & $\approx 0.170^{\mathrm{a}, \mathrm{c}}$ & $\begin{array}{c}\approx 100000^{c} \text { (Day } \\
20 \text { ) }\end{array}$ & n.a. & \\
\hline & & $12 / 12$ & $\begin{array}{c}120 \mu \mathrm{mol} \\
\text { photons } / \mathrm{m}^{2} / \mathrm{s} \text { at } 25^{\circ} \mathrm{C}\end{array}$ & $\approx 0.120^{\mathrm{a}, \mathrm{c}}$ & $\begin{array}{c}\approx 60000^{\circ} \text { (Day } \\
20 \text { ) }\end{array}$ & n.a. & \\
\hline & & $12 / 12$ & $\begin{array}{c}20 \mu \mathrm{mol} \text { photons } / \mathrm{m}^{2} / \mathrm{s} \\
\text { at } 28^{\circ} \mathrm{C}\end{array}$ & $\approx 0.070^{\mathrm{a}, \mathrm{c}}$ & $\begin{array}{c}\approx 15000^{\circ} \text { (Day } \\
15)\end{array}$ & n.a. & \\
\hline & & $12 / 12$ & $\begin{array}{c}40 \mu \mathrm{mol} \text { photons } / \mathrm{m}^{2} / \mathrm{s} \\
\text { at } 28^{\circ} \mathrm{C}\end{array}$ & $\approx 0.120^{a, c}$ & $\begin{array}{c}\approx 35000^{\circ} \text { (Day } \\
15)\end{array}$ & $\mathrm{OA} \approx 2.625^{\mathrm{b}, \mathrm{c}}$ & \\
\hline & & $12 / 12$ & $\begin{array}{c}80 \mu \mathrm{mol} \text { photons } / \mathrm{m}^{2} / \mathrm{s} \\
\text { at } 28^{\circ} \mathrm{C}\end{array}$ & $\approx 0.100^{a, c}$ & $\begin{array}{c}\approx 35000^{\circ} \text { (Day } \\
15)\end{array}$ & n.a. & \\
\hline & & $12 / 12$ & $\begin{array}{c}120 \mu \mathrm{mol} \\
\text { photons } / \mathrm{m}^{2} / \mathrm{s} \text { at } 28^{\circ} \mathrm{C}\end{array}$ & no growth & - & n.a. & \\
\hline \multirow{4}{*}{$\begin{array}{l}\text { P. hoffmannianum } \\
\text { (882a, Isolates } \\
\text { from Little } \\
\text { Lameshur Bay, } \\
\text { St John, US } \\
\text { Virgin Island) }\end{array}$} & \multirow{4}{*}{$\begin{array}{l}\text { Modified } \mathrm{K} \text { medium in seawater } \\
\text { (no Tris, Cu and Si); salinity } \\
36 \% ; 27^{\circ} \mathrm{C}\end{array}$} & $16 / 8$ & $2000 \operatorname{lux}$ & $\begin{array}{c}\approx 0.06-0.2^{\mathrm{C}} \\
\left(\max \text { at } 27^{\circ} \mathrm{C}\right)\end{array}$ & n.a. & $\begin{array}{l}\mathrm{OA} \approx 10-53.8^{\mathrm{C}} \\
\left(\mathrm{max} \text { at } 23^{\circ} \mathrm{C}\right)\end{array}$ & Morton et al., 1994 \\
\hline & & $16 / 8$ & 3000 lux & $\begin{array}{l}\approx 0.1-0.425^{\circ} \\
\left(\max \text { at } 29^{\circ} \mathrm{C}\right)\end{array}$ & n.a. & $\begin{array}{l}\mathrm{OA} \approx 11.3-38^{\circ} \\
\left(\max \text { at } 29^{\circ} \mathrm{C}\right)\end{array}$ & \\
\hline & & $16 / 8$ & $4000 \operatorname{lux}$ & $\begin{array}{l}\approx 0.07-0.425^{\circ} \\
\left(\max \text { at } 27^{\circ} \mathrm{C}\right)\end{array}$ & n.a. & $\begin{array}{c}\mathrm{OA} \approx 5-45^{\circ} \\
\left(\max \text { at } 29^{\circ} \mathrm{C}\right)\end{array}$ & \\
\hline & & $16 / 8$ & 5000 lux & $\begin{array}{l}\approx 0.08-0.53^{\circ} \\
\left(\max \text { at } 27^{\circ} \mathrm{C}\right)\end{array}$ & n.a. & $\begin{array}{c}\mathrm{OA} \approx 2.5-17.5^{\mathrm{C}} \\
\left(\max \text { at } 25 \text { and } 31^{\circ} \mathrm{C}\right)\end{array}$ & \\
\hline
\end{tabular}

n.a.: not available; ${ }^{a}$ The specific growth rate is calculated according to the following formula: $\mu=\left(\left(\ln N_{1}\right)-\ln \left(N_{0}\right)\right) /\left(t_{1}-t_{0}\right)$ where $N_{0}$ and $N_{1}$ are the cell density at time $t_{0}$ and $t_{1}$; ${ }^{b}$ The toxin level was determined at stationary phase; ${ }^{c}$ The data were estimated from data in the corresponding publication. 
TABLE 4 | Effect of salinity on the growth and toxin content of Prorocentrum spp.

\begin{tabular}{|c|c|c|c|c|c|c|}
\hline Strain & Culture & $\begin{array}{c}\text { Salinity } \\
(\%)\end{array}$ & $\begin{array}{c}\text { Growth rate } \\
(\mu / \text { day })\end{array}$ & $\begin{array}{l}\text { Maximum } \\
\text { cell density } \\
\text { (cells/mL) }\end{array}$ & $\begin{array}{c}\text { Toxin content } \\
\text { (pg/cell) }\end{array}$ & References \\
\hline \multirow[t]{3}{*}{$\begin{array}{l}\text { P. lima } \\
\text { (CCMP 2579) }\end{array}$} & \multirow{3}{*}{$\begin{array}{l}\mathrm{F} / 2 \text { medium; salinity } 30 \% \text {; } \\
25^{\circ} \mathrm{C} ; 100 \mu \mathrm{mol} \text { photons } / \mathrm{m}^{2} / \mathrm{s} \text {; } \\
\text { initial cell density } 5000 \mathrm{cells} / \mathrm{mL}\end{array}$} & 15 & n.a. & $\begin{array}{l}\approx 11500 \\
(\text { Day } 35)\end{array}$ & $\begin{array}{c}\mathrm{OA} \approx 1.0^{\mathrm{b}, \mathrm{c}} \\
\mathrm{DTX} 1 \approx 4.7^{\mathrm{b}, \mathrm{c}}\end{array}$ & \multirow[t]{3}{*}{$\begin{array}{l}\text { Wang et al., } \\
2015\end{array}$} \\
\hline & & 30 & n.a. & $\begin{array}{l}\approx 25000 \\
\text { (Day 35) }\end{array}$ & $\begin{array}{c}\mathrm{OA} \approx 1.8^{\mathrm{b}, \mathrm{c}} \\
\mathrm{DTX} 1 \approx 11.0^{\mathrm{b}, \mathrm{c}}\end{array}$ & \\
\hline & & 45 & n.a. & $\begin{array}{c}\approx 7000 \\
(\text { Day 35) }\end{array}$ & $\begin{array}{c}\mathrm{OA} \approx 3.0^{\mathrm{b}, \mathrm{c}} \\
\mathrm{DTX} 1 \approx 22.5^{\mathrm{b}, \mathrm{c}}\end{array}$ & \\
\hline \multirow{5}{*}{$\begin{array}{l}\text { P. hoffmannianum } \\
\text { (882a, Isolates } \\
\text { from Little } \\
\text { Lameshur Bay, } \\
\text { St John, US } \\
\text { Virgin Island) }\end{array}$} & \multirow{5}{*}{$\begin{array}{l}\text { Modified K medium in seawater } \\
\text { (no Tris, Cu and Si); salinity } \\
36 \% ; 27^{\circ} \mathrm{C} ; 3000 \text { lux; } 16 / 8 \mathrm{~h} \\
\text { light/dark cycle }\end{array}$} & 28 & $\approx 0.125^{\mathrm{c}}$ & n.a. & $\mathrm{OA} \approx 7.5^{\mathrm{c}}$ & \multirow{5}{*}{$\begin{array}{l}\text { Morton et al., } \\
1994\end{array}$} \\
\hline & & 31 & $\approx 0.135^{\mathrm{c}}$ & n.a. & $\mathrm{OA} \approx 4.5^{\mathrm{c}}$ & \\
\hline & & 34 & $\approx 0.220^{\mathrm{C}}$ & n.a. & $\mathrm{OA} \approx 3.2^{\mathrm{C}}$ & \\
\hline & & 37 & $\approx 0.160^{\mathrm{c}}$ & n.a. & $\mathrm{OA} \approx 3.5^{\mathrm{C}}$ & \\
\hline & & 40 & $\approx 0.135^{\mathrm{C}}$ & n.a. & $\mathrm{OA} \approx 4^{\mathrm{C}}$ & \\
\hline
\end{tabular}

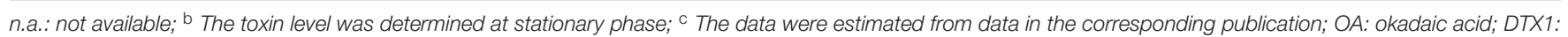
dinophysistoxin-1.

In routine medium recipes, the composition of trace metals has been formulized to meet the biochemical requirements of most dinoflagellates culture. Yet, in some cases, modifications are needed as each Prorocentrum spp. has its own distinctive growth requirements. Furthermore, trace metal composition may discourage or promote phycotoxin production by dinoflagellates (Rhodes et al., 2006; He et al., 2010). For example, Se at pM level induced an increase of $\mathrm{OA}$ ester quota per cell in P. lima by approximately 50\% (Rhodes et al., 2006). Gu et al. (2019) observed that an increase of $\mathrm{Cu}$ concentration (from 40 to 5039 nM) limited the growth of $P$. lima by $36 \%$ but increased the cellular OA concentration level 1.8 times ( $27 \mathrm{pg} / \mathrm{cell})$. The effects of trace metals on dinoflagellate culture and their implications for mass cultivation and bioactive metabolite production strategies require further exploration.

\section{Effect of Physical Factors}

Other than nutritional factors, physico-chemical factors such as light, salinity and temperature have been shown to impact on the growth and the phycotoxin production of dinoflagellates (Morton et al., 1994; López-Rosales et al., 2014; Tan and Ransangan, 2015; Wang et al., 2015).

\section{Light}

As with other photoautotrophic phytoplankton, growth of dinoflagellates is directly affected by the availability and irradiance of light. In general, artificial light is chosen to culture dinoflagellates as it provides better control of the light spectrum, irradiance and photoperiod (Table 3) (Morton et al., 1994; LópezRosales et al., 2014; Wang et al., 2015). Light-emitting diodes (LEDs) are an excellent alternative as they outperform fluorescent light. LEDs are adjustable to fit the design of the photobioreactors (PBRs), provide effective light without generating too much heat, are low-cost and non-toxic. A few studies have shown the potential of LEDs to culture dinoflagellates (Schulze et al., 2014; López-Rosales et al., 2016; Molina-Miras et al., 2018).

Light intensity and photoperiod influence not only the growth and cell cycle of Prorocentrum spp. but also their cellular phycotoxin composition (Table 3). In the study of López-Rosales et al. (2014) excessive light intensity led to the photoinhibition of $P$. belizeanum. Maximum growth of $P$. belizeanum was found at $40 \mu \mathrm{mol}$ photons $/ \mathrm{m}^{2} / \mathrm{s}$ regardless of the temperature $\left(18-28^{\circ} \mathrm{C}\right)$. P. hoffmannianum growth rate increased as the irradiance increased from 2000 lux $(0.20 \mu /$ day $)$ to 5000 lux $(0.53 \mu /$ day $)$. High phycotoxin production has been observed at low light intensity conditions. Intracellular content of OA by $P$. hoffmannianum was higher $(53.8 \mathrm{pg} / \mathrm{cell})$ at low light intensity (2000 lux) in comparison to higher light intensities (5000 lux; OA: 17.5 pg/cell). The most commonly used light/dark cycles to study phycotoxin production are $8 \mathrm{~h} / 16 \mathrm{~h}, 12 \mathrm{~h} / 12 \mathrm{~h}$ and $16 \mathrm{~h} / 8 \mathrm{~h}$. Wang et al. (2015) reported that the light duration is directly proportional to maximum cell density. Maximum cell density of $P$. lima increased from $8 \mathrm{~h}(\approx 11000$ cells $/ \mathrm{mL})$ to $12 \mathrm{~h}(\approx 25500$ cells $/ \mathrm{mL})$ and remained fairly constant after $16 \mathrm{~h}$ $(\approx 26000$ cells $/ \mathrm{mL})$ of light. However, cellular OA content was lower when the light exposure was increased up to $16 \mathrm{~h}$. Little is known about the relationship between phycotoxin production and photosynthesis that could be potentially associated to cell cycles (Pan et al., 1999). OA was suggested to be biosynthesized in the chloroplast (Zhou and Fritz, 1994) and stored in them or in peripheral vacuoles of $P$. lima (Barbier et al., 1999). Wang et al. (2015) indicated that both photosynthesis and dark respiration are needed for DSP toxin biosynthesis. Pan et al. (1999) indicated that DSP production was instigated by light. DSP toxin amount per cell (OA, OA C8-diol-ester, DTX1 and DTX4) increased soon after $P$. lima culture was exposed to light after a dark synchronization period (no changes during dark). Different DSP toxins were produced at different phase of the photocycle. A rise in DTX4 levels (initiated in the G1 to $S$ phases "morning") preceded an increase in the cellular content of OA and DTX1 (produced during S and G2 phases "afternoon") 3 to $6 \mathrm{~h}$ later. Based on the sequential pattern in the biosynthesis of DTX4, OA, OA-C8 diol-ester and DTX1, DTX4 was considered the precursor toxin in the biosynthetic cascade through a light-mediated stepwise enzymatic reactions. In Praptiwi (2014), light and dark cycle at low to medium 
TABLE 5 | Effect of temperature on the growth and toxin content of Prorocentrum spp.

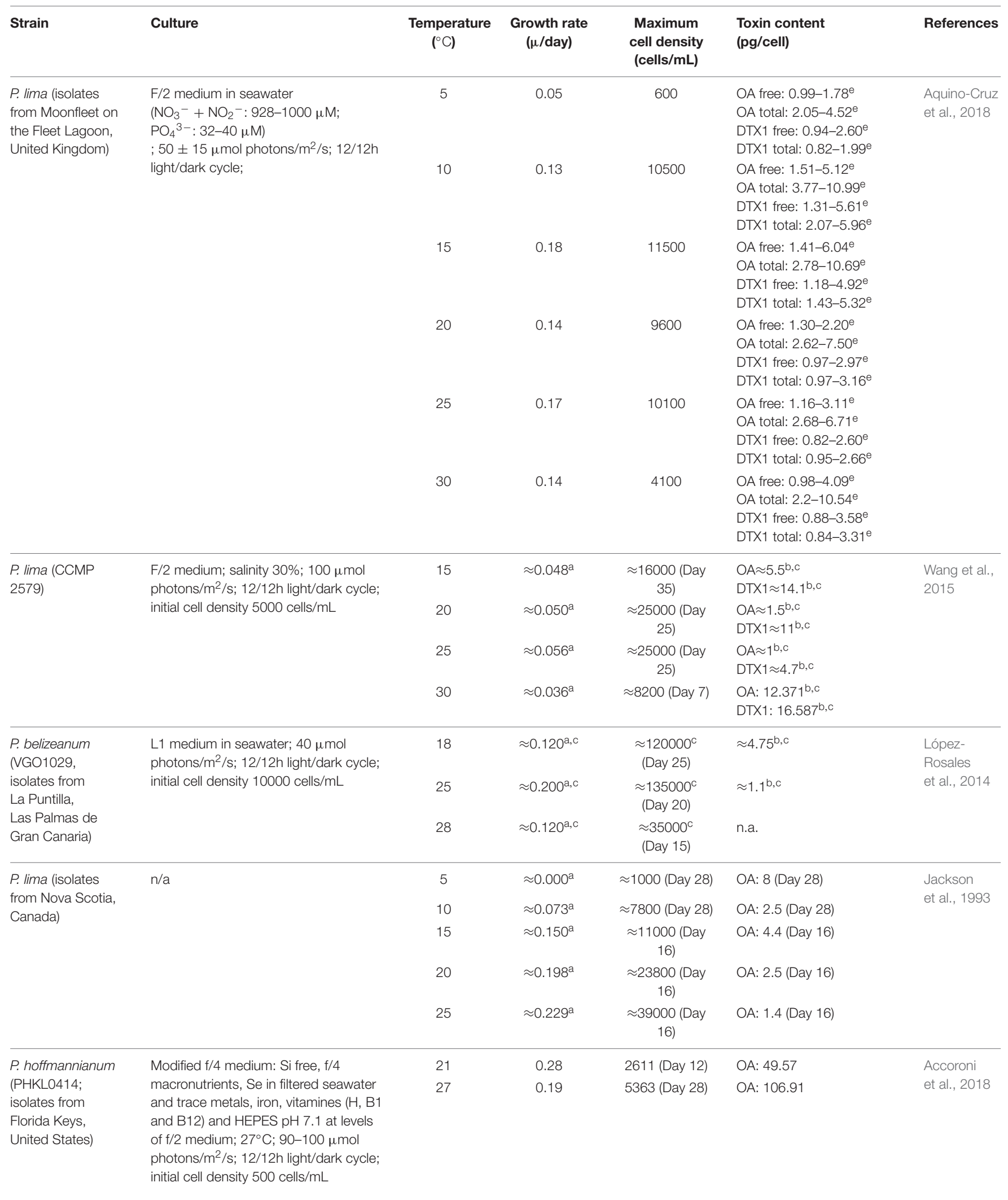

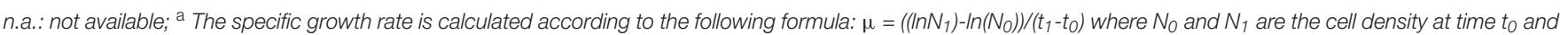
$t_{1} ;{ }^{b}$ The toxin level was determined at stationary phase; ${ }^{\mathrm{C}}$ The data were estimated from data in the corresponding publication; ${ }^{\mathrm{e}}$ Ranges consider lag, exponential and stationary growth phases; OA: okadaic acid; DTX1: dinophysistoxin-1. 
light intensities (25 and $50 \mu \mathrm{mol} / \mathrm{m}^{2} / \mathrm{s}$ ) were proved to have no substantial effect to the total yield of OA and DTX-1, when compared to cultures with continuous light exposure. It did, however, improved the low yield gained in cultures exposed to high light intensity $\left(95 \mu \mathrm{mol} / \mathrm{m}^{2} / \mathrm{s}\right.$ ), with approximately $36 \%$ increase for OA and $66 \%$ increase for DTX-1 when using 12 hours light and dark cycles compared to continuous cycle. Despite this, the study (Praptiwi, 2014) further found that these improvements in yields are still significantly lower than those obtained from cultures exposed to low and medium light intensities ( 25 and $50 \mu \mathrm{mol} / \mathrm{m}^{2} / \mathrm{s}$ ) at both continuous light and 12 hours light/dark cycles.

\section{Salinity}

Salinity influences biochemical processes in the cell such as nutrient uptake and transportation system inside cell (Hsieh et al., 2001). Variations in ion strength and ionic composition induces distinctive responses on the growth of dinoflagellates due to osmoregulation (Stefels, 2000). Growth tolerance under a range of salinities content appears to be different for each species and is attributed to its geographic origin (D'Ors et al., 2016; Monti-Birkenmeier et al., 2019).

In principle, changes in salinity could affect phycotoxin production and content, either by direct effect on the biosynthetic pathways, or indirectly, by modulating cell growth which in turn indirectly affect cellular phycotoxin content (Parkhill and Cembella, 1999; Aguilera-Belmonte et al., 2013). This is supported by evidence that despite the same salinity, phycotoxin production appeared to be variable throughout the growth phase; with a constant level during the exponential growth phase which starts to increase at early stationary phase (Parkhill and Cembella, 1999). However, it was argued by Lim et al. (2011) that phycotoxin production is not a result of salinity-dependentgrowth rate, but rather governed by cell osmoregulation and utilization of amino acid. Lim et al. (2011) further explained that evidence of this was shown in their results of toxin quota (Qt) obtained from the two lowest growth cultures at 5 and $35 \mathrm{psu}$, where much lower Qt observed in 5 psu than 35 psu.

Very few studies (Table 4) have dealt with the effect of salinity on growth rate and phycotoxin production by Prorocentrum spp. and they show contradictory results. In general growth rate decreased with salinity $(0.125 \mu$ /day at $28 \%$ and $0.105 \mu$ /day at $37 \%$; at $40 \%$ there was $0.120 \mu$ day) (Morton et al., 1994). The maximum cell density during the stationary phase also declined with increasing salinity (Wang et al., 2015). The cellular phycotoxin content in $P$. lima increased with increasing salinity. The Qt of OA increased from $\approx 1.0 \mathrm{pg} /$ cell to $\approx 3.0 \mathrm{pg} / \mathrm{cell}$ and DTX1 from $\approx 4.7 \mathrm{pg} /$ cell to $\approx 22.5 \mathrm{pg} /$ cell when the salinity was increased from 15 psu to 45 psu (Wang et al., 2015). On the other hand, cellular content of OA in P. hoffmannianum was inversely proportional to salinity content and growth rate (Morton et al., 1994). The maximum cellular OA level $(\approx 7.5$ $\mathrm{pg} /$ cell $)$ was reached at $28 \mathrm{psu}$ and the lowest $(\approx 3.2 \mathrm{pg} /$ cell $)$ was found at $34 \mathrm{psu}$ which coincided with the highest growth rate $(\approx 0.22 \mu)$. As the salinity was increased up to 40 psu the growth rate declined $(\approx 0.135 \mu)$ and the OA cellular content slightly increased up to $\approx 4.0 \mathrm{pg} /$ cell $)$. 


\section{Temperature}

Effects of temperature on growth and phycotoxin production of dinoflagellates appear to be similar to salinity, where each species has a different temperature range that enables optimum growth and bioactive compound production, which is a dependent on its original habitat (Koike et al., 1998; Aguilera-Belmonte et al., 2013). Benthic Prorocentrum spp. grow in tropical regions $\left(\approx 30^{\circ} \mathrm{C}\right)$, whereas epiphytic strains grow in colder waters. Hence, Prorocentrum spp. are able to adapt to a broad range of temperatures (Table 5). For instance P. lima isolated from temperate areas (Canada) can grow between 10 and $25^{\circ} \mathrm{C}$ (Jackson et al., 1993), whereas if it is a strain isolated from tropical regions (United States) it grows between 21 and $27^{\circ} \mathrm{C}$ (Accoroni et al., 2018). In particular, $25^{\circ} \mathrm{C}$ is the optimal growth temperature for P. lima and P. belizeanum (Jackson et al., 1993; López-Rosales et al., 2014; Wang et al., 2015; Aquino-Cruz et al., 2018). Morton et al. (1994) observed that $P$. hoffmannianum can tolerate temperatures between 21 and $36^{\circ} \mathrm{C}$, and gave $27^{\circ} \mathrm{C}$ as the optimal temperature.

Temperature has been found to influence growth, enzymatic activities and phycotoxin production in Prorocentrum spp. (López-Rosales et al., 2014; Lee et al., 2016).

Stress caused by changes in temperature promote phycotoxin production in Prorocentrum spp. Several studies have shown that maximum growth and cell density of Prorocentrum spp. obtained at specific temperatures were disassociated from maximum cellular phycotoxin levels (Jackson et al., 1993; López-Rosales et al., 2014; Wang et al., 2015; Aquino-Cruz et al., 2018) (Table 5). Disagreement on the influence that temperatures have above or below the optimal growth temperature to obtain the highest OA production have been published. For instance, temperatures above the optimal growth temperature of $P$. lima led to an increase of up to 12 times of the cellular OA level and up to 3.5 times of DTX1 (Wang et al., 2015) and up to 2.2 times of $\mathrm{OA}$ in P. hoffmannianum (Accoroni et al., 2018). In contrast, lower temperatures led to an increase of the cellular phycotoxin content of OA and DTX1 in P. lima and P. belizeanum (Jackson et al., 1993; López-Rosales et al., 2014; Aquino-Cruz et al., 2018). Extreme temperatures led to an increase of the cellular phycotoxin content although the relationship between temperature and phycotoxin production is not clear (Lee et al., 2016).

\section{MASS CULTIVATION OF DINOFLAGELLATES}

Early studies on secondary metabolites from dinoflagellates mostly attempted to understand how environmental and nutritional parameters affect the production of phycotoxins as a control strategy for HABs and their related health impacts. Nevertheless, the potential applications of these phycotoxins had shifted the interest to optimize their production at large scale.

For example, various attempts were carried out to find the most efficient way of producing OA for commercial demands. Total synthesis of OA was successfully demonstrated by a study (Ley et al., 1998) which assembled three chemical fragments in a convergent manner but this method is somewhat timeconsuming, requires many steps with low final yield, and may be impractical for a large-scale production. Another approach was to extract OA from natural blooms of microalgae in seawater by a large-scale pumping method, as shown in a study by Rundberget et al. (2007). Nevertheless, considering the small quantity of purified phycotoxin obtained (2.7 mg OA and $1.3 \mathrm{mg}$ DTX2 from $\approx 10250 \mathrm{~L}$ of seawater containing $\approx 2 \times 10^{4} \mathrm{cells} / \mathrm{L}$ of Dinophysis acuta), this method can be unsustainable as it consumes a lot of energy. The limitations of these approaches highlight the convenience and practicality of culturing organisms that produce $\mathrm{OA}$ and other bioactive compounds.

One of the biggest challenges in microalgae mass cultivation is to design and operate a cost-effective production system that allow the control over the growth rate and product yield. To address low product yields and upscaling the main parameters to control are nutritional and environmental factors, light availability and mass and heat transfer as well and efficient PBR design.

Traditionally, microalgae have been cultivated in shallow open PBRs using a raceway design and basic mixing. Unfortunately, they are not suitable for toxic microalgae as they pose a considerable risk for the ecosystem and are prone to contamination (Gallardo-Rodríguez et al., 2012). Conversely, closed PBRs allow more control over operating conditions and less chance of contamination. Various configurations of closed PBRs have been used for culturing dinoflagellates including carboys, stirred-tanks, airlift, bubble column and flat-panel type, with the last two the most typically used (Wang et al., 2015; López-Rosales et al., 2016; Molina-Miras et al., 2018). Selection of closed PBRs will depend on the characteristics of the culture organism, the desired product and other parameters such as capital and maintenance costs. The culture can be operated as batch, fed-batch, continuous, or semi-continuous modes. Continuous systems are attractive due to the possibility of maintain optimum growth and automatization however, they need constant supply of fresh media, and they are complex to install and run as well as being more expensive. In semicontinuous mode fresh media is introduced as required, at interval periods, using much less volume of media than the continuous system. However, to date only a few studies described the cultivation of $P$. lima to produce $\mathrm{OA}$ and all of them operate in batch mode (Table 6).

Light is one of the key parameters to enhance the productivity of phototropic microalgae. Light should be provided at the appropriate intensity, wavelength and duration (Carvalho et al., 2011). Light attenuation and/or mutual shading effects should be limited to ensure culture efficiency and lower energy consumption. It is necessary to design a PBR that has a high surface to volume ratio, and relatively low culture thickness (López-Rosales et al., 2014). Wang et al. (2015) designed a vertical flat bioreactor with an illumination system arranged at the bottom of the PBR. This design promoted the light energy absorption and utilization of benthic microalgae (Wang et al., 2015). Light and dark cycles allow the cells to perform photosynthesis during the light regime and respiration during the dark regime. An alternative to continuous light availability is 
intermittent and "flashing" light which have proven to efficiently promote photosynthesis rate (Acién Fernández et al., 2013) and increase the productivity of secondary metabolites from microalgae (Carvalho et al., 2011). LEDs have been satisfactory used in pilot-scale bubble column PBRs for dinoflagellate culturing (Schulze et al., 2014; López-Rosales et al., 2016; Molina-Miras et al., 2018).

Phototrophic microalgae produce oxygen and uptake carbon dioxide during photosynthesis altering the culture medium and the $\mathrm{pH}$. Therefore, it is essential to remove the produced oxygen from the medium and to supply enough $\mathrm{CO}_{2}$ as the carbon source (Posten, 2009). In addition, as benthic microalgae grow, they form lumps that attach to the bottom of the bioreactor forming dense mats and some strains (e.g., P. belizeanum) produce extracellular mucus that can cover the bottom of the reactor forming a thin layer (López-Rosales et al., 2014). Gas injection is essential to increase mass transfer but designing a suitable culture system for dinoflagellate production can be particularly difficult in ensuring that the cell viability is maintained. Dinoflagellates are extremely sensitive to fluid turbulence in the culture media (García Camacho et al., 2007) and most of them do not readily grow in closed PBRs due to this (Gallardo-Rodríguez et al., 2009, 2012). Small-scale turbulence may affect growth rate and morphology and excessive turbulence may result in flagella impairment due to physical damage (Berdalet et al., 2007; García Camacho et al., 2007; Wang and Lan, 2018). Not only the intensity of shear force should be taken into account but also its period and interval (Gibson and Thomas, 1995). Detrimental effects of shear stress include growth inhibition; increased time for recovery, reduced photosynthetic activity, disturbed cell cycles, production of reactive oxygen species (ROS), changes in the fluidity of the cell membrane and even cell lysis (García Camacho et al., 2007; Gallardo-Rodríguez et al., 2009; Gallardo Rodríguez et al., 2010). No adverse effects were observed when the specific energy dissipation rate was below $1 \mathrm{~cm}^{2} / \mathrm{s}^{3}$ (Berdalet et al., 2007). The mechanism of shear-induced damage on cells has been hypothesized in previous studies. It has been suggested that damage is linked to their enormous genomes packed in relatively small volumes, to the alteration of metabolic processes that induce the production of intracellular ROS, to the reduction of time-integrated light exposure of individual cells or to direct impact on the integrity of cellular organelles (Juhl et al., 2001; Garcia-Camacho et al., 2007; Gallardo-Rodríguez et al., 2009). Antioxidant agents and additives to alter the fluidity of the media have been proven useful at low concentrations to protect the cells against shear stress (Gallardo-Rodríguez et al., 2009, 2012; Gallardo Rodríguez et al., 2010). Despite this, fluid turbulence is an important aspect in a bioreactor to maintain mass transfer within the system. It ensures good mixing of nutrients and gas exchange, and prevents deposition of cells at the bottom of the reactor, as well as forcing the cells to move between light and dark

\section{REFERENCES}

Accoroni, S., Ceci, M., Tartaglione, L., Romagnoli, T., Campanelli, A., Marini, M., et al. (2018). Role of temperature and nutrients on the growth and toxin production of Prorocentrum hoffmannianum (Dinophyceae) from zones of the PBR. Overall, selecting the cultivation system should reflect on a design that is able to provide sufficient mass transfer whilst maintaining cell viability of the culture.

\section{CONCLUSION}

Despite the negative impact of phycotoxins from dinoflagellates on human health, they have shown valuable pharmacological and biotechnological potential. OA and its derivatives are needed for research studies mainly due to their activity as selective protein phosphatases inhibitors. Besides OA, DTXs and analogs, Prorocentrum spp. produce many other secondary metabolites such as prorocentrolides and formosalides with interesting bioactive potential that remain neglected. Limited quantities in the producer organism as well as the variability of these high value secondary metabolites content urge a suitable production method to ensure adequate and consistent supply. As chemical synthesis, harvesting of natural blooms and genetic engineering strategies currently have considerable limiting factors (e.g., complex, low yield, expensive and scalability issues) culturing has proven to be the most suitable process to obtain high quantities of phycotoxins. Considerable efforts have been made into culturing to enhance phycotoxin production by Prorocentrum spp. at lab scale but just a few studies have demonstrated significant increase in yields. Discrepancies observed among performance highlight the absence of generic culturing protocols (e.g., age, cell density of inoculum) to maximize the growth rate of Prorocentrum spp. and the production of valuable secondary metabolites. Some advances have been made on the design of PBRs, in terms of nutritional and operational factors, however a few technological limitations still need to be addressed to grow this extremely shear sensitive organisms.

\section{AUTHOR CONTRIBUTIONS}

DC-M: writing - original draft and writing - review and editing. RP: writing - review and editing. LL: writing - review and editing and conceptualization. CE: writing - review and editing, conceptualization, and funding acquisition. All authors contributed to the article and approved the submitted version.

\section{FUNDING}

The authors would like to thank the Biotechnology and Biological Sciences Research Council (BBSRC) [BB/S004211/1] for funding. This publication was developed from networking activities of Christine Edwards during COST Action CA18238 (Ocean4Biotech), supported by COST (European Cooperation in Science and Technology) program.

the Florida Keys. Harmful Algae 80, 140-148. doi: 10.1016/j.hal.2018. 11.005

Acién Fernández, F. G., Fernández Sevilla, J. M., and Molina Grima, E. (2013). Photobioreactors for the production of microalgae. Rev. Environ. Sci. Biottechnol. 12, 131-151. 
Aguilera-Belmonte, A., Inostroza, I., Carrillo, K. S., Franco, J. M., Riobó, P., and Gómez, P. I. (2013). The combined effect of salinity and temperature on the growth and toxin content of four Chilean strains of Alexandrium catenella (Whedon and Kofoid) Balech 1985 (Dinophyceae) isolated from an outbreak occurring in southern Chile in (2009). Harmful Algae 23, 55-59. doi: 10.1016/j. hal.2012.12.006

Ajuzie, C. C. (2007). Palatability and fatality of the dinoflagellate Prorocentrum lima to Artemia salina. J. Appl. Phycol. 19, 513-519. doi: 10.1007/s10811-0079164-9

Amar, M., Aráoz, R., Iorga, B. I., Yasumoto, T., Servent, D., and Molgó, J. (2018). Prorocentrolide-A from cultured Prorocentrum lima dinoflagellates collected in Japan blocks sub-types of nicotinic acetylcholine receptors. Toxins 10:97. doi: 10.3390/toxins10030097

Anderson, D. M., Cembella, A. D., and Hallegraeff, G. M. (2012). Progress in understanding harmful algal blooms: paradigm shifts and new technologies for research, monitoring, and management. Ann. Rev. Mar. Sci. 4, 143-176. doi: 10.1146/annurev-marine-120308-081121

Aquino-Cruz, A., Purdie, D. A., and Morris, S. (2018). Effect of increasing sea water temperature on the growth and toxin production of the benthic dinoflagellate Prorocentrum lima. Hydrobiologia 813, 103-122. doi: 10.1007/s10750-0183512-4

Armstrong, R. W., Beau, J. M., Cheon, S. H., Christ, W. J., Fujioka, H., Ham, W. H., et al. (1989). Total synthesis of palytoxin carboxylic acid and palytoxin amide. J. Am. Chem. Soc. 111, 7530-7533. doi: 10.1021/ja00201a038

Assunção, J., Guedes, A. C., and Malcata, F. X. (2017). Biotechnological and pharmacological applications of biotoxins and other bioactive molecules from dinoflagellates. Mar. Drugs 15, 393. doi: 10.3390/md15120393

Barbier, M., Amzil, Z., Mondeguer, F., Bhaud, Y., Soyer-Gobillard, M.-O., and Lassus, P. (1999). Okadaic acid and PP2A cellular immunolocalization in Prorocentrum lima (Dinophyceae). Phycologia 38, 41-46. doi: 10.2216/i00318884-38-1-41.1

Berdalet, E., Peters, F., Koumandou, V. L., Roldán, C., Guadayol, Ò, and Estrada, M. (2007). Species-specific physiological response of dinoflagellates to quantified small-scale turbulence. J. Phycol. 43, 965-977. doi: 10.1111/j.15298817.2007.00392.x

Bhaud, Y., Guillebault, D., Lennon, J. F., Defacque, H., Soyer-Gobillard, M. O., and Moreau, H. (2000). Morphology and behaviour of dinoflagellate chromosomes during the cell cycle and mitosis. J. Cell Sci. 113, 1231-1239. doi: 10.1242/jcs. 113.7.1231

Bialojan, C., and Takai, A. (1988). Inhibitory effect of a marine-sponge toxin, okadaic acid, on protein phosphatases. Specificity and kinetics. Biochem. J. 256, 283-290. doi: 10.1042/bj2560283

Bravo, I., Fernández, M. L., Ramilo, I., and Martıìnez, A. (2001). Toxin composition of the toxic dinoflagellate Prorocentrum lima isolated from different locations along the Galician coast (NW Spain). Toxicon 39, 1537-1545. doi: 10.1016/s0041-0101(01)00126-x

Brown, A. R., Lilley, M., Shutler, J., Lowe, C., Artioli, Y., Torres, R., et al. (2020). Assessing risks and mitigating impacts of harmful algal blooms on mariculture and marine fisheries. Rev. Aquacult. 12, 1663-1688.

Bruland, K. W., Donat, J. R., and Hutchins, D. A. (1991). Interactive influences of bioactive trace metals on biological production in oceanic waters. Limnol. Oceanogr. 36, 1555-1577. doi: 10.4319/lo.1991.36.8.1555

Camacho-Muñoz, D., Lawton, L. A., and Edwards, C. (2020). Degradation of okadaic acid in seawater by $\mathrm{UV} / \mathrm{TiO}_{2}$ photocatalysis - Proof of concept. Sci. Tot. Environ. 733:139346. doi: 10.1016/j.scitotenv.2020.139346

Carvalho, A. P., Silva, S. O., Baptista, J. M., and Malcata, F. X. (2011). Light requirements in microalgal photobioreactors: an overview of biophotonic aspects. Appl. Microbiol. Biotechnol. 89, 1275-1288. doi: 10.1007/s00253-0103047-8

Cassell, R. T., Chen, W., Thomas, S., Liu, L., and Rein, K. S. (2015). Brevetoxin, the dinoflagellate neurotoxin, localizes to thylakoid membranes and interacts with the light-harvesting complex II (LHCII) of photosystem II. ChemBioChem 16, 1060-1067. doi: 10.1002/cbic.201402669

Cembella, A. D. (2003). Chemical ecology of eukaryotic microalgae in marine ecosystems. Phycologia 42, 420-447. doi: 10.2216/i0031-8884-42-4-420.1

Cohen, P., Holmes, C. F. B., and Tsukitani, Y. (1990). Okadaic acid: a new probe for the study of cellular regulation. Trends Biochem. Sci. 15, 98-102. doi: 10.1016/ 0968-0004(90)90192-e
Cousseau, A., Siano, R., Probert, I., Bach, S., and Mehiri, M. (2020). "Marine dinoflagellates as a source of new bioactive structures," in Studies in Natural Products Chemistry, ed. R. Atta ur (Amsterdam: Elsevier), 125-171. doi: 10. 1016/b978-0-12-817905-5.00004-4

Crimmins, M. T., Ellis, J. M., Emmitte, K. A., Haile, P. A., McDougall, P. J., Parrish, J. D., et al. (2009). Enantioselective total synthesis of brevetoxin a: unified strategy for the B, E, G, and J subunits. Chem. Eur. J. 15, 9223-9234. doi: 10.1002/chem.200900776

Cruz, P. G., Norte, M., Creus, A. H., Fernández, J. J., and Daranas, A. H. (2013). Self-association of okadaic acid: Structural and pharmacological significance. Mar. Drugs 11, 1866-1877. doi: 10.3390/md11061866

D’Ors, A., Bartolomé, M. C., and Sánchez-Fortún, S. (2016). Repercussions of salinity changes and osmotic stress in marine phytoplankton species. Estuar. Coast. Shelf Sci. 175, 169-175. doi: 10.1016/j.ecss.2016.04.004

Dounay, A. B., Urbanek, R. A., Sabes, S. F., and Forsyth, C. J. (1999). Total synthesis of the marine natural product 7-deoxy-okadaic acid: A potent inhibitor of serine/threonine-specific protein phosphatases. Angewandte Chemie Int. Ed. 38, 2258-2262. doi: 10.1002/(sici)1521-3773(19990802)38:15<2258::aidanie2258>3.0.co;2-\#

Durán-Riveroll, L. M., Cembella, A. D., and Okolodkov, Y. B. (2019). A review on the biodiversity and biogeography of toxigenic benthic marine dinoflagellates of the coasts of Latin America. Front. Mar. Sci. 6:148. doi: 10.3389/fmars.2019. 00148

Farabegoli, F., Blanco, L., Rodríguez, L. P., Vieites, J. M., and Cabado, A. G. (2018). Phycotoxins in marine shellfish: origin, occurrence and effects on humans. Mar. Drugs 16:188. doi: 10.3390/md16060188

Fernández, J. J., Suárez-Gómez, B., Souto, M. L., and Norte, M. (2003). Identification of new okadaic acid derivatives from laboratory cultures of Prorocentrum lima. J. Nat. Prod. 66, 1294-1296. doi: $10.1021 / \mathrm{np030110u}$

Forsyth, C. J., Sabes, S. F., and Urbanek, R. A. (1997). An efficient total synthesis of okadaic acid. J. Am. Chem. Soc. 119, 8381-8382. doi: 10.1021/ja9715206

Fu, L.-1, Zhao, X.-Y., Ji, L.-D., and Xu, J. (2019). Okadaic acid (OA): Toxicity, detection and detoxification. Toxicon 160, 1-7. doi: 10.1016/j.toxicon.2018.12. 007

Fürstner, A., Flügge, S., Larionov, O., Takahashi, Y., Kubota, T., and Kobayashi, J. I. (2009). Total synthesis and biological evaluation of amphidinolide V and analogues. Chem. Eur. J. 15, 4011-4029. doi: 10.1002/chem.200802068

Gallardo Rodríguez, J. J., Sánchez Mirón, A., García Camacho, F., Cerón García, M. C., Belarbi, E. H., and Molina Grima, E. (2010). Culture of dinoflagellates in a fed-batch and continuous stirred-tank photobioreactors: Growth, oxidative stress and toxin production. Proc. Biochem. 45, 660-666. doi: 10.1016/j.procbio. 2009.12 .018

Gallardo-Rodríguez, J. J., Sánchez-Mirón, A., García-Camacho, F., Cerón-García, M. C., Belarbi, E. H., Chisti, Y., et al. (2009). Causes of shear sensitivity of the toxic dinoflagellate Protoceratium reticulatum. Biotechnol. Prog. 25, $792-800$.

Gallardo-Rodríguez, J., Sánchez-Mirón, A., García-Camacho, F., López-Rosales, L., Chisti, Y., and Molina-Grima, E. (2012). Bioactives from microalgal dinoflagellates. Biotechnol. Adv. 30, 1673-1684. doi: 10.1016/j.biotechadv.2012. 07.005

García Camacho, F., Gallardo Rodríguez, J. J., Sánchez Mirón, A., Cerón García, M. C., Belarbi, E. H., and Molina Grima, E. (2007). Determination of shear stress thresholds in toxic dinoflagellates cultured in shaken flasks: Implications in bioprocess engineering. Proc. Biochem. 42, 1506-1515. doi: 10.1016/j.procbio. 2007.08.001

Garcia-Camacho, F., Gallardo-Rodríguez, J., Sánchez-Mirón, A., Cerón-García, M. C., Belarbi, E. H., Chisti, Y., et al. (2007). Biotechnological significance of toxic marine dinoflagellates. Biotechnol. Adv. 25, 176-194. doi: 10.1016/j. biotechadv.2006.11.008

Gibson, C. H., and Thomas, W. H. (1995). Effects of turbulence intermittency on growth inhibition of a red tide dinoflagellate, Gonyaulax polyedra Stein. J. Geophys. Res. Oceans 100, 24841-24846. doi: 10.1029/95jc02256

Glibert, P. M., Wilkerson, F. P., Dugdale, R. C., Raven, J. A., Dupont, C. L., Leavitt, P. R., et al. (2016). Pluses and minuses of ammonium and nitrate uptake and assimilation by phytoplankton and implications for productivity and community composition, with emphasis on nitrogen-enriched conditions. Limnol. Oceanogr. 61, 165-197. doi: 10.1002/lno.10203 
Gómez, F. (2012). A quantitative review of the lifestyle, habitat and trophic diversity of dinoflagellates (Dinoflagellata, Alveolata). System. Biodivers. 10, 267-275. doi: 10.1080/14772000.2012.721021

Griffith, A. W., and Gobler, C. J. (2020). Harmful algal blooms: A climate change co-stressor in marine and freshwater ecosystems. Harmful Algae 91:101590. doi: 10.1016/j.hal.2019.03.008

Gu, S., Xiao, S.-W., Zheng, J.-W., Li, H.-Y., Liu, J.-S., and Yang, W.-D. (2019). $\mathrm{ABC}$ transporters in Prorocentrum lima and their expression under different environmental conditions including okadaic acid production. Mar. Drugs 17:259. doi: $10.3390 / \mathrm{md} 17050259$

Guillard, R. R. L., and Morton, S. L. (2003). "Culture Methods," in Manual on Harmful Marine Microalgae, eds G. M. Hallegraef, D. M. Anderson, and A. D. Cembella (Paris: UNESCO Publishing).

Guillebault, D., Sasorith, S., Derelle, E., Wurtz, J. M., Lozano, J. C., Bingham, S., et al. (2002). A new class of transcription initiation factors, intermediate between TATA box-binding proteins (TBPs) and TBP-like factors (TLFs), is present in the marine unicellular organism, the dinoflagellate Crypthecodinium cohnii. J. Biol. Chem. 277, 40881-40886. doi: 10.1074/jbc.m205624200

Hallegraeff, G. M. (2010). Ocean climate change, phytoplankton community responses, and harmful algal blooms: A formidable predictive challenge. J. Phycol. 46, 220-235. doi: 10.1111/j.1529-8817.2010.00815.x

Harrison, P. J., and Berges, J. A. (2005). "Marine culture media," in Algal Culturing Techniques, ed. R. Andersen (Florida, FL: Academic Press), 21-33. doi: 10.1016/ b978-012088426-1/50004-4

He, H., Chen, F., Li, H., Xiang, W., Li, Y., and Jiang, Y. (2010). Effect of iron on growth, biochemical composition and paralytic shellfish poisoning toxins production of Alexandrium tamarense. Harmful Algae 9, 98-104. doi: 10.1016/ j.hal.2009.08.006

Hinder, S. L., Hays, G. C., Brooks, C. J., Davies, A. P., Edwards, M., Walne, A. W., et al. (2011). Toxic marine microalgae and shellfish poisoning in the British isles: history, review of epidemiology, and future implications. Environ. Health 10:54.

Holmes, M. J., Lee, F. C., Khoo, H. W., and Teo, S. L. M. (2001). Production of 7-deoxy-okadaic acid by a new caledonian strain of Prorocentrum lima (Dinophyceae). J. Phycol. 37, 280-288. doi: 10.1046/j.1529-8817.2001. 037002280.x

Hoppenrath, M. (2017). Dinoflagellate taxonomy - a review and proposal of a revised classification. Mar. Biodivers. 47, 381-403. doi: 10.1007/s12526-0160471-8

Hoppenrath, M., Chomérat, N., Horiguchi, T., Schweikert, M., Nagahama, Y., and Murray, S. (2013). Taxonomy and phylogeny of the benthic Prorocentrum species (Dinophyceae)-A proposal and review. Harmful Algae 27, 1-28. doi: 10.1016/j.hal.2013.03.006

Hou, D.-Y., Liang, J.-J., Zou, C., Li, H.-Y., Liu, J.-S., and Yang, W.-D. (2016). MRP functional activity and character in the dinoflagellate Prorocentrum lima. J. Appl. Phycol. 28, 1667-1676. doi: 10.1007/s10811-015-0679-1

Hsieh, D. P. H., Wang, D., and Chang, G. H. (2001). Laboratory bioproduction of paralytic shellfish toxins in dinoflagellates. Adv. Appl. Microbiol. 49, 85-110. doi: 10.1016/s0065-2164(01)49010- 1

Hu, T., Curtis, J. M., Walter, J. A., and Wright, J. L. C. (1995). Identification of DTX-4, a new water-soluble phosphatase inhibitor from the toxic dinoflagellate Prorocentrum lima. J. Chem. Soc. Chem. Commun. 1995, 597-599. doi: 10.1039/ c39950000597

Hu, T., Marr, J., de Freitas, A. S. W., Quilliam, M. A., Walter, J. A., Wright, J. L. C., et al. (1992). New Diol Esters Isolated from Cultures of the Dinoflagellates Prorocentrum lima and Prorocentrum concavum. J. Nat. Prod. 55, 1631-1637. doi: 10.1021/np50089a011

Ianora, A., Bentley, M. G., Caldwell, G. S., Casotti, R., Cembella, A. D., EngströmÖst, J., et al. (2011). The relevance of marine chemical ecology to plankton and ecosystem function: An emerging field. Mar. Drugs 9, 1625-1648. doi: $10.3390 /$ md 9091625

Isobe, M., Ichikawa, Y., and Goto, T. (1986). Synthetic studies toward marine toxic polyethers [5] the total synthesis of okadaic acid. Tetrahedron Lett. 27, 963-966. doi: 10.1016/s0040-4039(00)84149-0

Jackson, A. E., Marr, J. C., and MacLachlan, J. L. (1993). "The production of diarrhetic shellfish toxins by an isolate of Prorocentrum lima from Nova Scotia, Canada," in 5th International conference on toxic marine phytoplankton, Toxic phytoplankton blooms in the sea, (Newport, RI: Elsevier), 513-518.
Jacob-Lopes, E., Scoparo, C. H. G., Lacerda, L. M. C. F., and Franco, T. T. (2009). Effect of light cycles (night/day) on $\mathrm{CO}_{2}$ fixation and biomass production by microalgae in photobioreactors. Chem. Eng. Process. 48, 306-310. doi: 10.1016/ j.cep.2008.04.007

James, K. J., Carey, B., O’Halloran, J., van Pelt, F. N. A. M., and ŠKrabÁKovÁ, Z. (2010). Shellfish toxicity: human health implications of marine algal toxins. Epidemiol. Infect. 138, 927-940. doi: 10.1017/s0950268810000853

Jia, Y., Gao, H., Tong, M., and Anderson, D. M. (2019). Cell cycle regulation of the mixotrophic dinoflagellate Dinophysis acuminata: Growth, photosynthetic efficiency and toxin production. Harmful Algae 89:101672. doi: 10.1016/j.hal. 2019.101672

Juhl, A. R., Trainer, V. L., and Latz, M. I. (2001). Effect of fluid shear and irradiance on population growth and cellular toxin content of the dinoflagellate Alexandrium fundyense. Limnol. Oceanogr. 46, 758-764. doi: 10.4319/lo.2001. 46.4.0758

Kantiani, L., Llorca, M., Sanchís, J., Farré, M., and Barceló, D. (2010). Emerging food contaminants: a review. Anal. Bioanal. Chem. 398, 2413-2427.

Kohli, G. S., John, U., Van Dolah, F. M., and Murray, S. A. (2016). Evolutionary distinctiveness of fatty acid and polyketide synthesis in eukaryotes. ISME J. 10, 1877-1890. doi: 10.1038/ismej.2015.263

Koike, K., Sato, S., Yamaji, M., Nagahama, Y., Kotaki, Y., Ogata, T., et al. (1998). Occurrence of okadaic acid-producing Prorocentrum lima on the Sanriku coast, Northern Japan. Toxicon 36, 2039-2042. doi: 10.1016/s0041-0101(98)00132-9

Lee, S., Hwang, B. S., Kim, H. S., Yih, W., Jeong, E. J., and Rho, J.-R. (2015). A New Diol Ester Derivative of dinophysistoxin-1 from cultures of Prorocentrum lima collected in South Korea. Bull. Korean Chem. Soc. 36, 395-398. doi: 10.1002/ bkcs. 10031

Lee, S., Yang, A. R., Yoo, Y. D., Jeong, E. J., and Rho, J.-R. (2019). Relative configurational assignment of 4-hydroxyprorocentrolide and prorocentrolide C isolated from a benthic dinoflagellate (Prorocentrum lima). J. Nat. Prod. 82, 1034-1039. doi: 10.1021/acs.jnatprod.8b00988

Lee, T. C. H., Chan, P. L., Xu, S. J. L., and Lee, F. W. F. (2020). Comparison of growth and toxicity responses between non-toxic and toxic strains of Prorocentrum hoffmannianum. Aquat. Biol. 29, 59-70. doi: 10.3354/ab00725

Lee, T. C.-H., Fong, F. L.-Y., Ho, K.-C., and Lee, F. W.-F. (2016). The mechanism of diarrhetic shellfish poisoning toxin production in Prorocentrum spp.: Physiological and molecular perspectives. Toxins 8:272. doi: 10.3390/ toxins 8100272

Ley, S. V., Humphries, A. C., Eick, H., Downham, R., Ross, A. R., Boyce, R. J., et al. (1998). Total synthesis of the protein phosphatase inhibitor okadaic acid. J. Chem. Soc. Perkin Trans. 1, 3907-3912.

Li, J., Li, M., Pan, J., Liang, J., Zhou, Y., and Wu, J. (2012). Identification of the okadaic acid-based toxin profile of a marine dinoflagellate strain Prorocentrum lima by LC-MS/MS and NMR spectroscopic data. J. Sep. Sci. 35, 782-789. doi: 10.1002/jssc.201100914

Li, L. X., Tang, J. Q., Yang, W. D., Liu, J. S., Zhang, J. J., and Li, H. Y. (2009). Studies on toxin production of Prorocentrum lima. Asian J. Ecotox. 4, 745-750.

Li, Y.-Y., Tian, X.-Q., Lu, Y.-N., Han, Q.-H., Ma, L.-Y., and Fan, C.-Q. (2020). Toxins and other chemical constituents from Prorocentrum lima. Biochem. System. Ecol. 89:104015. doi: 10.1016/j.bse.2020.104015

Lim, P. T., Leaw, C. P., Sato, S., Thuoc, C. V., Kobiyama, A., and Ogata, T. (2011). Effect of salinity on growth and toxin production of Alexandrium minutum isolated from a shrimp culture pond in northern Vietnam. J. Appl. Phycol. 23, 857-864. doi: 10.1007/s10811-010-9593-8

Lim, Z. F., Luo, Z., Lee, L. K., Hii, K. S., Teng, S. T., Chan, L. L., et al. (2019). Taxonomy and toxicity of Prorocentrum from Perhentian Islands (Malaysia), with a description of a non-toxigenic species Prorocentrum malayense sp. nov. (Dinophyceae). Harmful Algae 83, 95-108. doi: 10.1016/j.hal.2019.01.007

Lin, S. (2011). Genomic understanding of dinoflagellates. Res. Microbiol. 162, 551-569. doi: 10.1016/j.resmic.2011.04.006

Lin, S., Litaker, R. W., and Sunda, W. G. (2016). Phosphorus physiological ecology and molecular mechanisms in marine phytoplankton. J. Phycol. 52, 10-36. doi: $10.1111 /$ jpy. 12365

Lin, W.-R., Tan, S.-I., Hsiang, C.-C., Sung, P.-K., and Ng, I. S. (2019). Challenges and opportunity of recent genome editing and multi-omics in cyanobacteria and microalgae for biorefinery. Biores. Technol. 291:121932. doi: 10.1016/j. biortech.2019.121932 
López-Rosales, L., Gallardo-Rodríguez, J. J., Sánchez-Mirón, A., Cerón-García, M. D. C., Belarbi, E. H., García-Camacho, F., et al. (2014). Simultaneous effect of temperature and irradiance on growth and okadaic acid production from the marine dinoflagellate Prorocentrum belizeanum. Toxins 6, 229-253. doi: $10.3390 /$ toxins 6010229

López-Rosales, L., García-Camacho, F., Sánchez-Mirón, A., Martín Beato, E., Chisti, Y., and Molina Grima, E. (2016). Pilot-scale bubble column photobioreactor culture of a marine dinoflagellate microalga illuminated with light emission diodes. Biores. Technol. 216, 845-855. doi: 10.1016/j.biortech. 2016.06.027

Louzao, M. C., Fernández, D. A., Abal, P., Fraga, M., Vilariño, N., Vieytes, M. R., et al. (2015). Diarrhetic effect of okadaic acid could be related with its neuronal action: Changes in neuropeptide Y. Toxicol. Lett. 237, 151-160. doi: 10.1016/j. toxlet.2015.06.004

Lu, C.-K., and Chou, H.-N. (2002). Research of marine dinoflagellate bioactive compounds in Taiwan. Fish. Sci. 68, 1593-1596. doi: 10.2331/fishsci.68.sup2_ 1593

Lu, C.-K., Chen, Y.-M., Wang, S.-H., Wu, Y.-Y., and Cheng, Y.-M. (2009). Formosalides A and B, cytotoxic 17-membered ring macrolides from a marine dinoflagellate Prorocentrum sp. Tetrahedron Lett. 50, 1825-1827. doi: 10.1016/ j.tetlet.2009.02.013

Lu, C.-K., Chou, H.-N., Lee, C.-K., and Lee, T.-H. (2005). Prorocentin, a new polyketide from the marine dinoflagellate Prorocentrum lima. Org. Lett. 7, 3893-3896. doi: 10.1021/ol051300u

Luckas, B., Dahlmann, J., Erler, K., Gerdts, G., Wasmund, N., Hummert, C., et al. (2005). Overview of key phytoplankton toxins and their recent occurrence in the North and Baltic Seas. Environ. Toxicol. 20, 1-17. doi: 10.1002/tox. 20072

Luo, Z., Zhang, H., Krock, B., Lu, S., Yang, W., and Gu, H. (2017). Morphology, molecular phylogeny and okadaic acid production of epibenthic Prorocentrum (Dinophyceae) species from the northern South China Sea. Algal Res. 22, 14-30. doi: 10.1016/j.algal.2016.11.020

McEwan, M., Humayun, R., Slamovits, C. H., and Keeling, P. J. (2008). Nuclear genome sequence survey of the dinoflagellate Heterocapsa triquetra. J. Eukaryot. Microbiol. 55, 530-535. doi: 10.1111/j.1550-7408.2008.00357.x

McLachlan, J. L., Marr, J. C., Conlon-Keily, A., and Adamson, A. (1994). Effects of nitrogen concentration and cold temperature on DSP-toxin concentrations in the dinoflagellate Prorocentrum lima (prorocentrales, dinophyceae). Nat. Toxins 2, 263-270. doi: 10.1002/nt.2620020504

Meštrović, V., and Pavela-Vrančič, M. (2003). Inhibition of alkaline phosphatase activity by okadaic acid, a protein phosphatase inhibitor. Biochimie $85,647-650$. doi: 10.1016/s0300-9084(03)00135-4

Molgó, J., Marchot, P., Aráoz, R., Benoit, E., Iorga, B. I., Zakarian, A., et al. (2017). Cyclic imine toxins from dinoflagellates: a growing family of potent antagonists of the nicotinic acetylcholine receptors. J. Neurochem. 142, 41-51. doi: 10.1111/jnc. 13995

Molina-Miras, A., López-Rosales, L., Sánchez-Mirón, A., Cerón-García, M. C., Seoane-Parra, S., García-Camacho, F., et al. (2018). Long-term culture of the marine dinoflagellate microalga Amphidinium carterae in an indoor LEDlighted raceway photobioreactor: Production of carotenoids and fatty acids. Biores. Technol. 265, 257-267. doi: 10.1016/j.biortech.2018.05.104

Monti-Birkenmeier, M., Berden Zrimec, M., Drinovec, L., Beran, A., Zrimec, A., Cataletto, B., et al. (2019). Influence of salinity on growth and cell volume in three strains of Prorocentrum cordatum (Dinophyceae). Aquat. Biol. 28, 1-12. doi: $10.3354 / \mathrm{ab} 00704$

Moreira-González, A. R., Fernandes, L. F., Uchida, H., Uesugi, A., Suzuki, T., Chomérat, N., et al. (2019). Variations in morphology, growth, and toxicity among strains of the Prorocentrum lima species complex isolated from Cuba and Brazil. J. Appl. Phycol. 31, 519-532. doi: 10.1007/s10811-018-1587-y

Moreno Díaz de la Espina, S., Alverca, E., Cuadrado, A., and Franca, S. (2005). Organization of the genome and gene expression in a nuclear environment lacking histones and nucleosomes: the amazing dinoflagellates. Eur. J. Cell Biol. 84, 137-149. doi: 10.1016/j.ejcb.2005.01.002

Morton, S. L., Bomber, J. W., and Tindall, P. M. (1994). Environmental effects on the production of okadaic acid from Prorocentrum hoffmannianum Faust I. temperature, light, and salinity. J. Exp. Mar. Bio. Ecol. 178, 67-77. doi: 10. 1016/0022-0981(94)90225-9
Munday, R. (2013). Is protein phosphatase inhibition responsible for the toxic effects of okadaic acid in animals? Toxins 5, 267-285. doi: 10.3390/ toxins 5020267

Murakami, Y., Oshima, Y., and Yasumoto, T. (1982). Identification of okadaic acid as a toxic component of a marine dinoflagellate Prorocentrum lima. Nippon Suisan Gakkaishi 48, 69-72. doi: 10.2331/suisan.48.69

Nascimento, S. M., Purdie, D. A., and Morris, S. (2005). Morphology, toxin composition and pigment content of Prorocentrum lima strains isolated from a coastal lagoon in southern UK. Toxicon 45, 633-649. doi: 10.1016/j.toxicon. 2004.12.023

Ng, I.-S., Keskin, B. B., and Tan, S.-I. (2020). A critical review of genome editing and synthetic biology applications in metabolic engineering of microalgae and cyanobacteria. Biotechnol. J. 15:1900228. doi: 10.1002/biot.201900228

Nicholas, G. M., and Phillips, A. J. (2006). Marine natural products: synthetic aspects. Nat. Prod. Reports 23, 79-99. doi: 10.1039/b501014b

Nicolaou, K. C., Frederick, M. O., Loizidou, E. Z., Petrovic, G., Cole, K. P., Koftis, T. V., et al. (2006). Second-generation total synthesis of azaspiracids-1, -2 , and -3. Chem. Asian J. 1, 245-263. doi: 10.1002/asia.200600059

Nishimura, T., Uchida, H., Noguchi, R., Oikawa, H., Suzuki, T., Funaki, H., et al. (2020). Abundance of the benthic dinoflagellate Prorocentrum and the diversity, distribution, and diarrhetic shellfish toxin production of Prorocentrum lima complex and P. caipirignum in Japan. Harmful Algae 96:101687. doi: 10.1016/j. hal.2019.101687

Norte, M., Padilla, A., Fernández, J. J., and Souto, M. L. (1994). Structural determination and biosynthetic origin of two ester derivatives of okadaic acid isolated from Prorocentrum lima. Tetrahedron 50, 9175-9180. doi: 10.1016/ s0040-4020(01)85383-7

O'Connor, P. D., and Brimble, M. A. (2007). Synthesis of macrocyclic shellfish toxins containing spiroimine moieties. Nat. Prod. Rep. 24, 869-885. doi: 10. 1039/b700307m

Orchard, M. J., Humphries, S., Schuech, R., and Menden-Deuer, S. (2016). The influence of viscosity on the motility and sensory ability of the dinoflagellate Heterocapsa triquetra. J. Plankton Res. 38, 1062-1076. doi: 10.1093/plankt/ fbw004

Pan, L., Chen, J., Shen, H., He, X., Li, G., Song, X., et al. (2017). Profiling of extracellular toxins associated with diarrhetic shellifish poison in Prorocentrum lima culture medium by high-performance liquid chromatography coupled with mass spectrometry. Toxins 9:308. doi: 10.3390/toxins9100308

Pan, Y., Cembella, A. D., and Quilliam, M. A. (1999). Cell cycle and toxin production in the benthic dinoflagellate Prorocentrum lima. Mar. Bio. 134, 541-549. doi: 10.1007/s002270050569

Pang, Y., Fang, C., Twiner, M. J., Miles, C. O., and Forsyth, C. J. (2011). Total synthesis of dinophysistoxin-2 and 2-epi-dinophysistoxin-2 and their PPase inhibition. Angew. Chem. Int. Ed. 50, 7631-7635. doi: 10.1002/anie.201101741

Park, S., Nguyen, T. H. T., and Jin, E. (2019). Improving lipid production by strain development in microalgae: Strategies, challenges and perspectives. Biores. Technol. 292:121953. doi: 10.1016/j.biortech.2019.121953

Parkhill, J.-P., and Cembella, A. D. (1999). Effects of salinity, light and inorganic nitrogen on growth and toxigenicity of the marine dinoflagellate Alexandrium tamarense from northeastern Canada. J. Plankton Res. 21, 939-955. doi: 10. 1093/plankt/21.5.939

Paz, B., Daranas, A. H., Cruz, P. G., Franco, J. M., Pizarro, G., Souto, M. L., et al. (2007). Characterisation of okadaic acid related toxins by liquid chromatography coupled with mass spectrometry. Toxicon 50, 225-235. doi: 10.1016/j.toxicon.2007.03.015

Perez, R., Liu, L., Lopez, J., An, T., and Rein, K. S. (2008). Diverse bacterial PKS sequences derived from okadaic acid-producing dinoflagellates. Mar. Drugs 6, 164-179. doi: 10.3390/md20080009

Pistocchi, R., Guerrini, F., Pezzolesi, L., Riccardi, M., Vanucci, S., Ciminiello, P., et al. (2012). Toxin levels and profiles in microalgae from the north-western adriatic sea-15 years of studies on cultured species. Mar. Drugs 10, 140-162. doi: $10.3390 / \mathrm{md} 10010140$

Posten, C. (2009). Design principles of photo-bioreactors for cultivation of microalgae. Eng. Life Sci. 9, 165-177. doi: 10.1002/elsc.200900003

Praptiwi, R. A. (2014). Optimisation of high value metabolite production from benthic marine dinoflagellate Prorocentrum lima. Ph. D. thesis. United Kingdom: Robert Gordon University. 
Qian, Z.-J., Kang, K.-H., and Ryu, B. (2015). "Microalgae-derived toxic compounds," in Handbook of Marine Microalgae, ed. S.-K. Kim (Boston: Academic Press), 527-537. doi: 10.1016/b978-0-12-800776-1.00035-2

Raven, J. A., Evans, M. C. W., and Korb, R. E. (1999). The role of trace metals in photosynthetic electron transport in $\mathrm{O}_{2}$-evolving organisms. Photosynth. Res. 60, 111-150.

Rhodes, L. L., and Syhre, M. (1995). Okadaic acid production by a New Zealand Prorocentrum lima isolate. N Z. J. Mar. Freshw. Res. 29, 367-370. doi: 10.1080/ 00288330.1995.9516671

Rhodes, L., Selwood, A., McNabb, P., Briggs, L., Adamson, J., van Ginkel, R., et al. (2006). Trace metal effects on the production of biotoxins by microalgae. Afr. J. Mar. Sci. 28, 393-397. doi: 10.2989/18142320609504185

Rill, R. L., Livolant, F., Aldrich, H. C., and Davidson, M. W. (1989). Electron microscopy of liquid crystalline DNA: direct evidence for cholesteric-like organization of DNA in dinoflagellate chromosomes. Chromosoma 98, 280286. doi: 10.1007/bf00327314

Rodríguez, F., Riobó, P., Crespín, G. D., Daranas, A. H., de Vera, C. R., Norte, M., et al. (2018). The toxic benthic dinoflagellate Prorocentrum maculosum Faust is a synonym of Prorocentrum hoffmannianum Faust. Harmful Algae 78, 1-8. doi: 10.1016/j.hal.2018.06.009

Rundberget, T., Sandvik, M., Larsen, K., Pizarro, G. M., Reguera, B., Castberg, T., et al. (2007). Extraction of microalgal toxins by large-scale pumping of seawater in Spain and Norway, and isolation of okadaic acid and dinophysistoxin-2. Toxicon 50, 960-970. doi: 10.1016/j.toxicon.2007. 07.003

Schmitz, F. J., and Yasumoto, T. (1991). The 1990 United States-Japan seminar on bioorganic marine chemistry, Meeting Report. J. Nat. Prod. 54, 1469-1490. doi: 10.1021/np50078a001

Schulze, P. S. C., Barreira, L. A., Pereira, H. G. C., Perales, J. A., and Varela, J. C. S. (2014). Light emitting diodes (LEDs) applied to microalgal production. Trends Biotechnol. 32, 422-430. doi: 10.1016/j.tibtech.2014.06.001

Selander, E., Thor, P., Toth, G., and Pavia, H. (2006). Copepods induce paralytic shellfish toxin production in marine dinoflagellates. Proc. Biol. Sci. 273, 16731680. doi: $10.1098 / \mathrm{rspb} .2006 .3502$

Shikata, T., Kitatsuji, S., Abe, K., Onitsuka, G., Matsubara, T., Nakayama, N., et al. (2020). Vertical distribution of a harmful red-tide dinoflagellate, Karenia mikimotoi, at the decline stage of blooms. J. Sea Res. 165:101960. doi: 10.1016/j. seares.2020.101960

Smayda, T. J. (1997). Harmful algal blooms: Their ecophysiology and general relevance to phytoplankton blooms in the sea. Limnol. Oceanogr. 42, 11371153. doi: 10.4319/lo.1997.42.5_part_2.1137

Smayda, T. J. (2010). Adaptations and selection of harmful and other dinoflagellate species in upwelling systems. 2. Motility and migratory behaviour. Prog. Oceanogr. 85, 71-91. doi: 10.1016/j.pocean.2010.02.005

Smayda, T. J., and Reynolds, C. S. (2003). Strategies of marine dinoflagellate survival and some rules of assembly. J. Sea Res. 49, 95-106. doi: 10.1016/s13851101(02)00219-8

Stefels, J. (2000). Physiological aspects of the production and conversion of DMSP in marine algae and higher plants. J. Sea Res. 43, 183-197. doi: 10.1016/s13851101(00)00030-7

Stivala, C. E., Gu, Z., Smith, L. L., and Zakarian, A. (2012). Studies toward the Synthesis of Spirolide C: Exploration into the Formation of the 23-Membered All-Carbon Macrocyclic Framework. Org. Lett. 14, 804-807. doi: 10.1021/ ol203342e

Suárez-Gómez, B., Souto, M. L., Cruz, P. G., Fernández, J. J., and Norte, M. (2005). New targets in diarrhetic shellfish poisoning control. J. Nat. Prod. 68, 596-599. doi: $10.1021 / \mathrm{np} 040183 \mathrm{t}$

Suárez-Gómez, B., Souto, M. L., Norte, M., and Fernández, J. J. (2001). Isolation and structural determination of DTX-6, a new okadaic acid derivative. J. Nat. Prod. 64, 1363-1364. doi: 10.1021/np0100486

Sunda, W. (2012). Feedback interactions between trace metal nutrients and phytoplankton in the ocean. Front. Microbiol. 3:204. doi: 10.3389/fmicb.2012. 00204

Tan, K. S., and Ransangan, J. (2015). "Factors influencing the toxicity, detoxification and biotransformation of paralytic shellfish toxins," in Reviews of Environmental Contamination and Toxicology, Vol. 235, ed. D. M. Whitacre (Berlin: Springer International Publishing), 1-25. doi: 10.1007/978-3-31910861-2_1
Tang, E. P. Y. (1996). Why do dinoflagellates have lower growth rates? J. Phycol. 32, 80-84. doi: 10.1111/j.0022-3646.1996.00080.x

Tarazona-Janampa, U. I., Cembella, A. D., Pelayo-Zárate, M. C., Pajares, S., Márquez-Valdelamar, L. M., Okolodkov, Y. B., et al. (2020). Associated bacteria and their effects on growth and toxigenicity of the dinoflagellate Prorocentrum lima species complex from epibenthic substrates along Mexican coasts. Front. Mar. Sci. 7:569. doi: 10.3389/fmars.2020.00569

Tian, C., Doblin, M. A., Dafforn, K. A., Johnston, E. L., Pei, H., and Hu, W. (2018). Dinoflagellate cyst abundance is positively correlated to sediment organic carbon in Sydney Harbour and Botany Bay, NSW, Australia. Environ. Sci. Pollut. Res. 25, 5808-5821. doi: 10.1007/s11356-017-0886-1

Torigoe, K. (1990). Structure and biosynthesis of bioactive substances produced by the dinoflagellate Prorocentrum lima. Ph. D. thesis. Sendai: Tohoku University.

Torigoe, K., Murata, M., Yasumoto, T., and Iwashita, T. (1988). Prorocentrolide, a toxic nitrogenous macrocycle from a marine dinoflagellate, Prorocentrum lima. J. Am. Chem. Soc. 110, 7876-7877. doi: 10.1021/ja00231a048

Twiner, M. J., Doucette, G. J., Pang, Y., Fang, C., Forsyth, C. J., and Miles, C. O. (2016). Structure-activity relationship studies using natural and synthetic okadaic acid/dinophysistoxin toxins. Mar. Drugs 14:207. doi: 10.3390/ md14110207

Usami, Y. (2009). Recent synthetic studies leading to structural revisions of marine natural products. Mar. Drugs 7, 314-330. doi: 10.3390/md7030314

Valdiglesias, V., Prego-Faraldo, M. V., Pásaro, E., Méndez, J., and Laffon, B. (2013). Okadaic acid: more than a diarrheic toxin. Mar. Drugs 11, 4328-4349. doi: 10.3390/md11114328

Valot, G., Mailhol, D., Regens, C. S., O’Malley, D. P., Godineau, E., Takikawa, H., et al. (2015). Concise total syntheses of amphidinolides C and F. Chem. Eur. J. 21, 2398-2408. doi: 10.1002/chem.201405790

Vanucci, S., Guerrini, F., Milandri, A., and Pistocchi, R. (2010). Effects of different levels of $\mathrm{N}$ - and P-deficiency on cell yield, okadaic acid, DTX-1, protein and carbohydrate dynamics in the benthic dinoflagellate Prorocentrum lima. Harmful Algae 9, 590-599. doi: 10.1016/j.hal.2010.04.009

Varkitzi, I., Pagou, K., Granéli, E., Hatzianestis, I., Pyrgaki, C., Pavlidou, A., et al. (2010). Unbalanced N:P ratios and nutrient stress controlling growth and toxin production of the harmful dinoflagellate Prorocentrum lima (Ehrenberg) Dodge. Harmful Algae 9, 304-311. doi: 10.1016/j.hal.2009.12.001

Varkitzi, I., Pagou, K., Pyrgaki, C., and Hatzianestis, I. (2017). A biomass upscale system for the marine dinoflagellate Prorocentrum lima and the production of bioactive lipophilic toxins. Int. J. Appl. Sci. Biotechnol. 5, 479-485. doi: 10.3126/ ijasbt.v5i4.18561

Verma, A., Barua, A., Ruvindy, R., Savela, H., Ajani, P. A., and Murray, S. A. (2019). The genetic basis of toxin biosynthesis in dinoflagellates. Microorganisms 7:222. doi: 10.3390/microorganisms7080222

Vilariño, N., Louzao, M. C., Abal, P., Cagide, E., Carrera, C., Vieytes, M. R., et al. (2018). Human poisoning from marine toxins: unknowns for optimal consumer protection. Toxins 10:324. doi: 10.3390/toxins 10080324

Wang, C., and Lan, C. Q. (2018). Effects of shear stress on microalgae - A review. Biotechnol. Adv. 36, 986-1002. doi: 10.1016/j.biotechadv.2018.03.001

Wang, S., Chen, J., Li, Z., Wang, Y., Fu, B., Han, X., et al. (2015). Cultivation of the benthic microalga Prorocentrum lima for the production of diarrhetic shellfish poisoning toxins in a vertical flat photobioreactor. Biores. Technol. 179, 243-248. doi: 10.1016/j.biortech.2014.12.019

Wilde, V. L., Morris, J. C., and Phillips, A. J. (2012). "Marine natural products synthesis," in Handbook of Marine Natural Products, eds E. Fattorusso, W. H. Gerwick, and O. Taglialatela-Scafati (Dordrecht: Springer), 601-673. doi: 10. 1007/978-90-481-3834-0_11

Wisecaver, J. H., and Hackett, J. D. (2011). Dinoflagellate genome evolution. Ann. Rev. Microbiol. 65, 369-387. doi: 10.1146/annurev-micro-090110-10 2841

Yang, A. R., Lee, S., Yoo, Y. D., Kim, H. S., Jeong, E. J., and Rho, J.-R. (2017). Limaol: A polyketide from the benthic marine dinoflagellate Prorocentrum lima. J. Nat. Prod. 80, 1688-1692. doi: 10.1021/acs.jnatprod.7b00127

Yang, W. D., Zhong, N., Liu, J. S., Zhang, J. L., and He, Y. (2008). Effects of different phosphorus sources on the growth and toxin production of Prorocentrum lima. J. Environ. Sci. 29, 2760-2765.

Yasumoto, T., Murata, M., Lee, J., and Torigoe, K. (1989). "Polyether toxins produced by dinoflagellates," in Mycotoxins and Phycotoxins '88: A Collection 
of Invited Papers Presented at the Seventh International IUPAC Symposium on Mycotoxins and Phycotoxins, eds S. Natori, K. Hasimoto, and Y. Ueno (Amsterdam: Elsevier), 375-382.

Yasumoto, T., Seino, N., Murakami, Y., and Murata, M. (1987). Toxins produced by benthic dinoflagellates. Biol. Bull. 172, 128-131. doi: 10.2307/1541612

Zhong, N., Yang, W., Liu, J., Zhang, J., and He, Y. (2008). Effects of different nitrogen sources on the growth and toxin production of Prorocentrum lima. Acta Sci. Circumst. 28, 1186-1191.

Zhou, J., and Fritz, L. (1994). Okadaic acid antibody localizes to chloroplasts in the DSP-toxin-producing dinoflagellates Prorocentrum lima and Prorocentrum maculosum. Phycologia 33, 455-461. doi: 10.2216/10031-8884-33-6-455.1
Conflict of Interest: The authors declare that the research was conducted in the absence of any commercial or financial relationships that could be construed as a potential conflict of interest.

Copyright $\odot 2021$ Camacho-Muñoz, Praptiwi, Lawton and Edwards. This is an open-access article distributed under the terms of the Creative Commons Attribution License (CC BY). The use, distribution or reproduction in other forums is permitted, provided the original author(s) and the copyright owner(s) are credited and that the original publication in this journal is cited, in accordance with accepted academic practice. No use, distribution or reproduction is permitted which does not comply with these terms. 